JPL Publication 87-6

$10-6 \%$

85245

$P-1$,

\title{
Approximating the Linear Quadratic Optimal Control Law for Hereditary Systems With Delays in the Control
}

Mark H. Milman

(NASA-CR-181161) APPROXIHATING THE LINEAR

N87-25813

QUADRATIC OPT IHAL CONTROL LAW FOR HEEEDITABY

SYSTEMS WITH DELAYS IN THE CONTROL (Jet

Propulsion Lab.) $70 \mathrm{p}$ Avail: NTIS HC

A $04 / M F$ AO 1

CSCL $12 \mathrm{~A} \quad \mathrm{G} 3 / 64$

Unclas

0085245

March 15, 1987

\section{N/SA}

National Aeronautics and

Space Administration

Jet Propulsion Laboratory

California Institute of Technology

Pasadena, California 
JPL Publication 87-6

\section{Approximating the Linear Quadratic Optimal Control Law for Hereditary Systems With Delays in the Control}

Mark H. Milman

March 15, 1987

\section{N/SA}

National Aeronautics and

Space Administration

Jet Propulsion Laboratory

California Institute of Technology

Pasadena, California 
The research described in this publication was carried out by the Jet Propulsion Laboratory, California Institute of Technology, under a contract with the National Aeronautics and Space Administration.

Reference herein to any specific commercial product, process, or service by trade name, trademark, manufacturer, or otherwise, does not constitute or imply its endorsement by the United States Government or the Jet Propulsion Laboratory. California Institute of Technology. 


\section{ABSTRACT}

The fundamental control synthesis issue of establishing a priori convergence rates of approximation schemes for feedback controllers for a class of distributed parameter systems is addressed within the context of hereditary systems. Specifically, a factorization approach is presented for deriving approximations to the optimal feedback gains for the linear regulator-quadratic cost problem associated with time-varying functional differential equations with control delays. The approach is based on a discretization of the state penalty which leads to a simple structure for the feedback control law. General propẹties of the Volterra factors of Hilbert-Schmidt operators are then used to obtain convergence results for the controls, trajectories and feedback kernels. Two algorithms are derived from the basic approximation scheme, including a fast algorithm, in the time-invariant case. A numerical example is also considered. 
CONTENTS

1. INTRODUCTION. . . . . . . . . . . . . . . . . . . . . . . . 1

2. PRELIMINARIES . . . . . . . . . . . . . . . . . . . 4 4

3. CONVERGENCE RESULTS . . . . . . . . . . . . . . . . . . 14

4. APPLICATIONS. . . . . . . . . . . . . . . . . . . . 31

5. ALGORITHMS. . . . . . . . . . . . . . . . . . . . . . . 42

CONCLUDING REMARKS. . . . . . . . . . . . . . . . . . . 60

FOOTNOTES . . . . . . . . . . . . . . . . . 61

ACKNOWLEDGEMENT . . . . . . . . . . . . . . . 62

REFERENCES. . . . . . . . . . . . . . . . . . 62 
1. INTRODUCTION. This report represents a first application of the control synthesis technique based on factorization methods developed in [24]. The main significance of the results contained herein stems from our ability to obtain a detailed analysis of a priori convergence rates for numerical approximation of feedback controllers for a class of distributed parameter systems. Although in the past 15 years much work has been done in developing approximation schemes for various distributed controllers, the important issue of convergence rates has been largely neglected. This is due primarily to the fact that it is a difficult problem, and control researchers have been content with the first order assessment regarding whether or not their approximation scheme converges at all (in certain correct senses). In this report convergence rates are established in the context of a challenging class of problems that include control delay terms. The underlying methods are of particular importance since they more tightly connect the modeling and control synthesis problems, and hence, have application to other distributed parameter systems.

The particular problem we address is the computation of the optimal feedback gain for the finite time linear regulator quadratic cost problem for systems governed by retarded functional differential equations (RFDE) with control delays. Feedback control laws for these systems have been previously derived for both the finite and infinite time problems in several articles under various hypotheses (see for example [6], [13], [14], [17], [24], [25]). A common approach in several of these articles is to relate the RFDE with control delays to an evolution equation in an appropriate state space. The feedback control law then arises in the familiar form as a solution to an operator Riccati equation. However, when point delays appear in the control, an unbounded input operator results and this operator then appears in the quadratic term of the Riccati equation. The presence of this unbounded term would appear to complicate any analysis of approximation schemes for the feedback gains.

The approach in [24] utilizing factorization theory [11], [22], allows us to circumvent these difficulties. The interest here is to further pursue the approach of [24] to the problem of approximating the optimal feedback kernel and deriving convergence estimates for the approximations, and the resulting controls and trajectories. (We note some other applications of factorization include for example, filtering and smoothing of nonstationary processes over a finite interval [15], [16], inverse problems in the spectral theory of differential operators [8], [18], solutions to two point boundary value problems $[21]$, and solutions to Fredholm equations of the first 
and second kinds, [11].) Although this specific problem has not to this author's knowledge been treated in the literature, several articles (e.g., [6], [10], [19]) have considered the approximation problem without control delays.

The approach in each of these articles involves expressing the RFDE as an evolution equation in the state space $\mathrm{R}^{\mathrm{N}} \mathrm{xL}_{2}$, and then approximating the resulting dynamical system. Delfour [5] discretizes in both the spatial and time variables, while Kunisch [19] and Gibson [10] discretize in only the spatial variable. Delfour considers the time-varying problem and obtains weak convergence of the solutions to the approximating Riccati equations. In [10] and [19] the open-loop semigroup is first approximated by discretizing the history space, and then the approximation theory of [9] is used for subsequent convergence analysis. This analysis is based on exploiting the relationship developed in [9] between the open-loop semigroup and the Riccati equation defining the feedback law. Open loop semigroup approximations have been derived in [2], [3]. By careful considerations involving the adjoint semigroup together with properties deduced from the finite dimensionality of the control space, Gibson was able to demonstrate strong convergence of the approximating Riccati operators. When coupled with the finite dimensionality of the input space, this leads to uniform convergence of the feedback operators. This is a significant result with respect to control implementation, since with uniform convergence the optimality of the approximating feedback law is independent of the state.

The convergence analysis presented in [10] and [19] depends heavily on the fact that the control map has finite rank. This condition does r.jt hold in the control delay problem and straightforward extensions of these convergence results to the control delay case are not apparent. However, the form of the feedback kernel derived in [24] remains amenable to approximation and convergence analysis regardless of the presence of control delays. The reason for this is that control delays do not present any complications in the open loop formulation of the optimal control law and that the relationship between open loop and closed loop is somewhat transparent in the approach of [24] - the feedback kernel is derived from linear operators involving the fundamental matrix of the RFDE and the solution to a certain factorization problem associated with the fundamental matrix. Convergence questions for approximations to the feedback gain then reduce to corresponding questions regarding convergence of solutions to 
related factorization problems. (We note that the integral Riccati equations of [9] sre also equivalent to certain factorization problems [23], so that the convergence analysis in [10] and [19] can also be performed within a factorization context.)

It will be shown that if the cost on the state in the regulator problem is a discrete sum with no integral term, then the associated factorization problem is solved by matrix inversion, and the exact feedback kernel can be defined in rerms of the fundamental matrix solution, quadrature, and the solutions to finite dimensional linear equations. (This form of the solution generalizes a result of Manitius [20] for the problem with terminal state penalty and no control delays.) Thus an approximation scheme for the problem containing an integral state penalty term can be developed by approximating this term by quadrature and solving exectly for the feedback kernel of the resulting discretized state cost problem. Using this approach together with factorization arguments we will be able to establish $O(1 / n) L_{\infty}$ - convergence for the approximate feedback kernels in the time-varying control delay case.

This result is (analytically) somewhat sharper than Gibson's in that the $L_{\infty}$ convergence of the kernels applies on the square as well as the diagonal, and also that a priori rates can be provided. The principal reason this sharfer result can be obtained is that we exploit the fact that only a computable piece of the semigroup - that part contributed by the fundamental matrix solution - is required to define the feedback kernel. Thus it is never necessary to consider the more difficult problem of approximating the entire semigroup. We now briefly outline the organization of the report.

In Section 2 the necessary mathematical preliminaries are developed and discussed. Because the approach does not follow along a Riccati synthesis of the solution, we will recapitulate in this section portions of the discussion in [24] relevant to the present application - particularly certain aspects of the Volterra factorization and how they apply to the RFDE control problem.

Section 3 contains the $\mathrm{I}_{\infty}$ - convergenca results for the feedback kernels, controls, and trajectories. Instead of considering specific quadrature schemes approximating the state cost, all the results are proved with respect to sequence of Borel measures satisfying certain convergence hypotheses. The key tool of this section is a 
factorization lemma which asserts that the factorization problem is well-posed (in an appropriate sense) in the space of integral operators with essentially bounded kernels.

In Section 4, the explicit form of the optimal feedback kemel associated with discrete state cost is derived. The resulting approximation scheme developed from the cost discretizations is then used as an analytical tool to obtain further results regarding the feedback kernel. Bor example, using essentially matrix manipulations, a Wiener-Hopf integral equation for the optimal feedback kernel is derived which is shown to be the control delay generalization of the Wiener-Hopf equation Manitius [20] had previously derived for the feedback kernel via a maximum principle.

In Sectivn 5 two algorithms representing implementation. of the basic approximation scheme of Section 4 are derived. In the time-invariant case a fast algorithm is derived by exploiting the near Toeplitz structure of the system of equations that defines the feedback kernel. A simple numerical example is also presented.

2. PRELIMINARIES. Let $[-T, T]$ denoto a closed and boundod interval in the real 1 ine with $I \geq 0$ and $T>0$, and 1 et $\sum$ denote the class of Borel subsets of [-I, T]. For an exbitrary Banach spece $Y,|y|$ will denote the norm of an element $g \in Y, B(Y, Z)$ vill denote the space of bounded 1 inear maps from $Y$ into another Banach space $Z$, and for brevity re vite $B(Y)$ for $B(Y, Y)$. Subscripts will sometimes be attached to the norm of an element to remove any ambiguities that aigh arise due to the fact that sereral different topologies will be used in the report. The notation $A^{*}$ (respectively $A^{\prime}$ ) will be used to denote the adjoint (transpose) of an operator (matrix).

In the sequel tho Banach space of contingons functions $C\left([-I, T], R^{N}\right)$ will be denoted $I$, the Hilbert space $L_{2}\left([-I, T], R^{M}\right)$ w11 be denoted $D$, and $H$ $\checkmark 111$ denote the Hilbert space $L_{2}\left([-r, T], R^{N}\right)$. Now define the resolution of the Identity $E: \sum \rightarrow B(D)$ by autiplication by the charecteristic function, 1.0. $[E(\omega) n](t)=X(\omega)(t) \mathrm{B}(t)(X(\omega)(t)=0$ if $t \omega, x(\omega)(t)=1$ if $t e \omega)$, and let $P^{t}$ denote the family of projections $E([-r, t])$. The complementary family, 
$I-P^{t}$, $i 11$ be denoted $P_{t}$. Note that $P^{t}$ is strongly continuous,

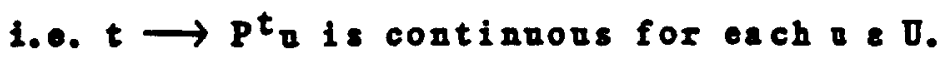

In this section ve sha11 review some of the results in [24] pertaining to the 1 inear regulator problem with dynamics

$$
\begin{array}{ll}
\dot{x}(t)=\int_{-x}^{0} d_{\theta} n(t, \theta) x(t+\theta)+\left(B P_{0} x\right)(t) & t \geq 0 \\
x(t)=\delta(t) & t \in[-x, 0]
\end{array}
$$

and quadratic cost functional,

$$
J(x, x)=\int_{-I}^{T}\langle x(s), Q(s) x(s)\rangle d \mu(s)+\int_{-I}^{T}|n(s)|^{2} d s
$$

In (2.1), we assune that $\delta(t)$ is contineons, $I(\cdot)$ \&, $u \in D, B \varepsilon B(D, B)$ and $P_{0}$ is the projection $E([O, T])$. (It rould not affect subsoguent convergenco analysis of the feedback kernels to allor an arbitrary projection $P_{t_{0}} t_{0}$ : [r. $T$, in place of $P_{0^{\circ}}$ The cholce $t_{0}=0$ reflects the problem formulation in vhich control policies cannot be implemented antil timet $=0$.) The only constraint we impose on Bat this time is that it be carsal, i.e.. for each $t \in[-r, T]$, if $a_{1}=a_{2}$ s.e. on $[0, t]$ then $\left(B n_{1}\right)(s)=\left(B a_{2}\right)(s)$ for a.e. s $\leq t$. Tho antix ralued function $\eta$ is assumedmessurabe on $R \times R$ and is normalized so that $\eta(t, \theta)=0$ for $\theta \leq 0$ and $\eta(t, \theta)=\eta(t,-r)$ for $\theta \leq-$ r. It 1 s further assued that $\eta(t, \cdot)$ is 1 of $t$ continuors for.each $t$ and there exists fanction m $L_{1}(0, T)$ sach that

$$
|\operatorname{Var} \eta(t, \cdot)| \leq m(t)
$$

where $|\cdot|$ denotes any natrix norm. In the cost (2.2). pdenotes an arbitrary positive regular Borel measure on $[-x, T]$, and $Q(\cdot)$ is Borel weasurable with

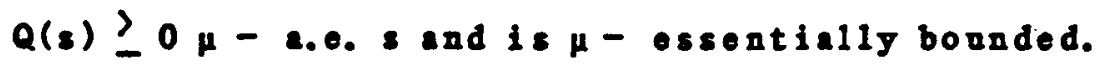


It is convenient at this time to introdnce some operators associated with the optimization problem (2.1) - (2.2). Te define:

$$
\begin{aligned}
& L \in B(X) ; L x: t \rightarrow \begin{cases}0 & t \in[-x, 0] \\
\int_{0}^{t} \int_{-I}^{0} d_{\theta} \eta(s, \theta) x(s+\theta) d s t \geq 0,\end{cases} \\
& F \in B(U, X) ; F n: t \rightarrow \int_{-T}^{t} F(t, s) v(s) d s .
\end{aligned}
$$

and the adjoint-1ike $F^{\#}$,

$$
F^{\#} \varepsilon B(X, \delta) ; F^{\#} \geq t \rightarrow \int_{t}^{T} F^{\prime}(s, t) Q(s) x(s) d \mu(s),
$$

Where $F(t, s)=\left[P_{0} B^{*} Y^{\prime}\left(t, 0^{-}\right)\right]^{\prime}(s)$ and $Y(t, s)$ is the fundamental aatrix solution of the homogeneous problem (see [12]). $Y(\bullet, \cdot)$ satisfies the Volterra oquation

$$
Y(t, s)= \begin{cases}I-\int_{s}^{t} Y(t, \sigma) \eta(\sigma, s-\sigma) d \sigma & s \leq t \\ 0 & s>t .\end{cases}
$$

and the solution to $(2.1)$ can be realized as

$$
\begin{aligned}
x(t) & =I(t, 0) \alpha(0)+\int_{-x}^{0} d_{\beta} \int_{0}^{t} I(t, \sigma) \eta(\sigma, \beta-\sigma) d \sigma \phi(\beta) \\
& +\int_{0}^{t} Y(t, \sigma)\left(B P_{0} \nabla\right)(\sigma) d \sigma .
\end{aligned}
$$


Also, sup $|Y(t, s)|<-, Y(t, s)$ is absolutely continoons for $t$ ? for each s, and $I(t, \cdot)$ is of bounded variation for each $t$. We note from the definition of $F(t, s)$ that $F(t, s)=0$ for $s, 0$. Hence, FPo $=F$ and $P_{0} F^{\#}=F^{\#}$

Using completing the squares argument, the open loop control 12 for (2.1) - (2.2) can be easily derived in terms of the operators dofined above.

Theored 2.1. The optinal control î for the regulator problen (2.1) - (2.2) is

$$
\hat{\mathbf{u}}=\mathbf{u} \tilde{\boldsymbol{\delta}}
$$

where $\mathrm{M} \varepsilon \mathrm{B}(\mathrm{X}, \mathrm{U}), 6 \in \mathrm{X}$,

$$
\begin{aligned}
& X=-\left(I+F^{\# F}\right)^{-1} F^{\#}(I-L)^{-1}, \\
& \tau(t)= \begin{cases}\delta(0) & t \geq 0 \\
\delta(t) & t \in[-I, 0] .\end{cases}
\end{aligned}
$$

Proof [24].

In [24] the foedback control $1 \mathrm{at}$ for (2.1) - (2.2) was derived from the open loop control above using actorization approach. In an effort to motivate this approsch and aske the report somerhat more self-contained, we will briefly retrace some of the stops in [24] leading to the feedback 1 an.

To sketch how the factorization idess arise in the foedback control synthosis, we will consider for convenience the case $\mu$ = Lebesgre measure, $Q(\cdot)=I, U=B$, and $B=$ the 1 dentity operator. Osing (2.7) we can then wite (2.1) $-(2.2)$ as

$$
\ln \langle x, x\rangle+\langle n, n\rangle
$$


subject to.

$$
x=f+T
$$

where $\langle\cdot, \cdot\rangle$ is the inner product on 0 (we can also take $I=U$ in this formalation), $T \& B(U)$ is the Bilbert-Schmidt operator

$$
\operatorname{Tz}: t \rightarrow \int_{0}^{t} I(t, s) \mathrm{u}(s) d s .
$$

and $f$ is the forcing term in $(2.8)$ resulting from the initial condition $\phi$

$$
f(t)=Y(t, 0)+\sigma(0) \int_{-I}^{0} d_{\beta} \int_{0}^{t} Y(t, \sigma) \eta(\sigma, \beta-\sigma) d \sigma \sigma(\beta) .
$$

Standard arguments then give the optimal control solntion $\hat{u}$ as

$$
\hat{z}=-\left(I+T^{*} T\right)^{-1} T^{*} f
$$

If Wo now consider (2.9) - (2.10) With the nodification that a $P_{\text {fl, }}$ $t \geq 0$, and roplace $f$ by an axbitrary forcing teraft the solution to this modifled problem is realized as

$$
\hat{\mathbf{u}}_{t}=-\left(\mathbf{I}+\mathbf{P}_{\mathbf{t}} \mathbf{T}^{*} \mathbf{T P _ { t }}\right)^{-1} \mathbf{P}_{\mathbf{t}} \mathbf{T}^{*} \mathbf{f}_{t}
$$

Using a principle of optimality (see [24]) it can be shorn that the choice $f_{t}=P_{t}\left[T^{t} \hat{t}+f\right]$ in the modified problem leads to $\hat{u}_{t}=P_{t} \hat{u}$ for each $t$. Thus, given partition of $[-x, T],-I=t_{0}<t_{1}<\cdots t_{n}=T$, it follors that

$$
\hat{z}=\sum E\left(\omega_{i}\right)\left[I+P_{t_{1}} T^{*} \operatorname{TP}_{t_{i}}\right\}^{-1} P_{t_{1}} T^{*} f_{t_{i}}
$$

$\omega_{i}=\left[t_{1}, t_{1+1}\right]$. Next, exanining the term $P_{t_{i}} T_{f_{t_{1}}}$ it is ovident that its values are determinod by $P_{t_{i}} f_{t_{i}}$ And for $\geq \geq t_{i}$, 


$$
\begin{aligned}
f_{t_{1}(s)} & =I(s, 0) \partial(0)+\int_{-I}^{0} d_{\beta}\left[\int_{0}^{s} I(s, \alpha) \eta(\alpha, \beta-\alpha) d \alpha\right\} \alpha(\beta) \\
& +\int_{0}^{t} I(s, a) \hat{u}(\alpha) .
\end{aligned}
$$

But tho bove is recognized as the solution to

$$
\begin{array}{ll}
\dot{x}(s)=\int_{-x}^{0} d_{\theta} \eta(s, \theta) x(s+\theta), & \leq t \\
x(s)=\hat{x}(s) & t-x \leq s \leq t,
\end{array}
$$

There $\hat{z}(s)$ is the optimal trejectory. The variation of constants formola then implies for $s$ ? $t_{i}$.

$$
f_{t_{i}}(s)=Y\left(s, t_{i}\right) \hat{x}\left(t_{i}\right)+\int_{t_{i}-x}^{t_{i}} \alpha_{\beta}\left\{\int_{t_{i}}^{s} Y(s, \alpha) \eta(\alpha, \beta-\alpha) d \alpha\right] \hat{I}(\beta) .
$$

Thos the optimel control in $(2.11)$ et time $t \cdot\left[t_{1}+t_{1+1}\right]_{\text {only }}$ eses the

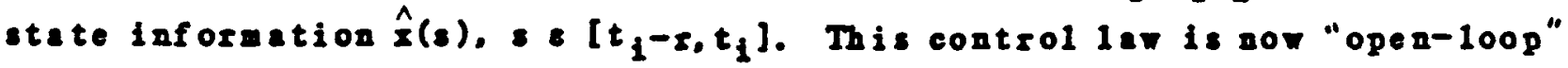
just on each sobinterval [ $t_{1}, t_{i+1}$ ]. The 1 imit as the mosh of the partitions in (2.11) tends to zero would hopefully produce tho foedbeck solution. This is indeed the case (ef [24]), and al though we will not go into all of the specifics regarding the 1 imiting procedure, we wil briefly discuss the very mech related notion of the projection integral ([11]).

Osing the same notations as bofore, let $G:[-x, T] \rightarrow B(D)$. Assume that $G$ is strongly continuous, 1.e. $t \rightarrow G(t)$ d is continuous for each $u$. Nor let $I: B(U)$ be Bilbort-Schmidt operator and consider Riemann sum of the form 


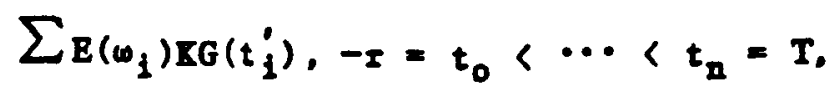

$\omega_{i}=\left[t_{i}, t_{i+1}\right]$, and $t_{i}^{\prime}=\omega_{i}$. These sums can be shown to converge in the operator norm as the mesh of the partitions tends to zero[11]. This 1 imit is represented by the projection integral

$$
\int \operatorname{dErG}(t) \text {, }
$$

and it can further be shown [11] that

$$
\left|\int \operatorname{aExG}(t)\right|_{\text {HS }} \leq|\mathbf{E}|_{\text {HS }} \sup |G(t)| \text {. }
$$

Bere $|\cdot|_{\text {HS }}$ denotes the Hilbert-Schmidt norm.

Tro important projection integrals on the space $\mathscr{K}$ of Hilbert-Schmidt ans in $B(U)$ are obtained from the seloctions $G_{+}(t)=P^{t}$ and $G_{-}(t)=P_{t}$ in (2.12). The resulting mappings $P_{ \pm}$'

$$
\mathbf{p}_{ \pm}(\mathbf{x})=\int_{\operatorname{dEKG}}(t)
$$

are bounded projections on $K_{\text {. If }}$. $R\left(p_{ \pm}\right)$we say that $I$ is causal (anticassi). The elements of $R\left(p_{ \pm}\right)$are quasinilpotents. In the sequel we shal1 also wite $K_{ \pm}$for $P_{ \pm}(X)$. Note also that $P^{t} D$ and $P_{t}$ are lavariant subspaces of $P_{\mp}(K)$, respectively.

We note that in the space $\mathrm{O}_{\text {a }}$ Hilbert-Schmidt map $\mathrm{K}$ is necossarily an integral operator with kernel, say $\mathbf{I}(t, s)$. In this case $p_{ \pm}(X)$ are simply the Volterra operators

$$
\begin{aligned}
& p_{+}(X) v: t \rightarrow \int_{-\tau}^{t}[(t, s) u(s) d s, \\
& p_{-}(I) v: t \rightarrow \int_{t}^{T}[(t, s) u(s) d s .
\end{aligned}
$$


With this bit of backgrond we can now state the basic factorization results thet 111 be used in the soquel.

Theorem 2.2. (Gohberg-Krein). Let $\mathbf{X}$. Then there exist anique operators $X_{ \pm} \in R\left(p_{ \pm}\right)$such that

$$
I+I=\left(I+I_{-}\right)\left(I+I_{+}\right)
$$

if and only if $\left(I+P_{t}\left(P_{t}\right)\right.$ is invertible for each $t \in[-I, T]$. Farthermore, N$=(I+I)^{-1}-I$ is given by the projoction integral

$$
\nabla_{-}=-\int \operatorname{dEKG}(t)
$$

with

$$
G(t)=P_{t}\left(I+P_{t}\left[P_{t}\right)^{-1}\right.
$$

The decomposition of $(I+I)$ into the product in (2.14) is called the Volterra or special (right) factorization of $I$ + and we vill sometimes

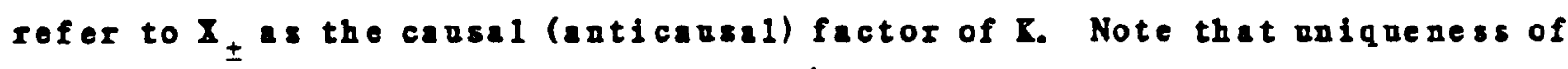
the factorization implies that $X_{-}=\left(X_{+}\right)^{*}$ when $X_{\text {is }}$ self-adjoint.

Tro results that will be nseful in subsequent convergence analysis are stated as corollaries below.

Corollary 2.3. Suppose $K$ e K' 1 self-adjoint ith

$$
\operatorname{sip}_{t}\left|\left(I+P_{t} \operatorname{LP}_{t}\right)^{-1}\right| \leq k
$$

Then $I+I$ hes the factorization $(2.14)$ and $\nabla_{ \pm}=\left(I+I_{ \pm}\right)^{-1}-I$ satisfy

$$
\left|\mathbf{I T}_{ \pm}\right|_{\mathrm{BS}} \leq \mathbf{z}|\mathbf{x}|_{\mathrm{BS}}
$$

Corol1ะry 2.4 . Lot $\mathbf{I}_{\mathrm{i}}, K_{,} 1=1,2$ be self-adjoint with

$$
\operatorname{sip}_{t}\left|\left(I+P_{t} \mathbf{R}_{i} P_{t}\right)^{-1}\right| \leq \mathbf{k}_{1}
$$


Let $\nabla_{i}^{*}=\left(I+x_{i}^{*}\right)^{-1}-I$ where $x_{i}^{*}$ denotes

the enticausal factor of $\mathbf{r}_{i}$. Then

$$
\left|\nabla_{1}^{*}-\nabla_{2}^{*}\right|_{\text {HS }} \leq \underset{i \neq j}{\min } k_{i}\left|x_{1}-z_{2}\right|_{\text {HS }}\left\{1+k_{j}\left|x_{j}\right|_{\text {HS }}\right\}
$$

Proof. Let $G_{1}(t)=P_{t}\left(I+P_{t} K_{i} P_{t}\right)^{-1}$. Then

$$
\begin{aligned}
& \left|\nabla_{1}^{*}-\nabla_{2}^{*}\right|_{\mathrm{HS}} \leq\left|\int \mathrm{dER}_{1}\left(G_{1}-G_{2}\right)\right|_{\mathrm{HS}}+\left|\int \mathrm{dE}\left(\mathbf{R}_{1}-\mathbf{K}_{2}\right) G_{2}\right|_{\mathrm{HS}} \\
& \leq\left|x_{1}\right|_{\text {BS }}\left|x_{1}-x_{2}\right|_{\text {HS }} k_{1} k_{2}+\left|x_{1}-\mathbf{z}_{2}\right|_{\text {HS }} k_{2} \\
& =k_{2}\left|\mathbf{z}_{1}-\mathbf{K}_{2}\right|_{\mathrm{BS}}\left\{1+\left|\mathrm{K}_{1}\right|_{\mathrm{BS}} \mathbf{k}_{1}\right\}
\end{aligned}
$$

Symmetry of the argument $\mathbf{i}$ th respect to $\mathbf{K}_{1}$ and $\mathbf{Z}_{2}$ gives the result./I

Returning nor to the control solution in (2.11), it can be shoran that the Riemann sums

$$
\sum E\left(\omega_{i}\right)\left(I+P_{t_{1}} T^{*} T P_{t_{1}}\right)^{-1} P_{t_{1}} T^{*}
$$

converge to the projection integral

$$
\int d E\left(I+I^{*}\right)^{-1} T^{*}
$$

Where $X$ is the causal factor of $T^{*} T, 1.0$.

$$
I+T^{*} T=\left(I+\mathbf{X}^{*}\right)(I+I)
$$

Nor given an element $h$ e $L_{2}([-x, T], 0)$ it is further possible (see [23]) to attach meaning to the expression

$$
-\int d B\left(I+x^{*}\right)^{-1} T^{*} h
$$

12 
2s a Iimit of $s \mathrm{~mm}$

$$
\sum_{i=1}^{n} E\left(\omega_{i, n}\right)\left(I+I^{*}\right)^{-1} T^{*} h_{i, n}
$$

Where the sequence of simple functions

$$
h_{n}=\sum x\left(w_{i, n}\right)(t) h_{i, n}
$$

converges in $L_{2}([-I, T], \delta)$ to h. The oxpression (2.15) is precisely $\hat{u}$ when $h(t)$ is chosen via the principle of optinality, $h(t)=P_{t}\left[T t^{t} \hat{u}+f\right]$ (cf $(2.11))$.

Now we proceed to define the feedback solution to (2.1) - (2.2). This follows from aerification of hypotheses and the evaluation of (2.15), with $h$ chosen above. Complete details can be found in [24].

Define the Hilbert space $H_{\mu}=L_{2}\left([-r, T], R^{N} ; \mu\right)$ as the space of $\mu$ - square integrable functions on $[-I, T]$-ith values in $R^{N}$. It is evident that the aep $Q: B_{\mu} \rightarrow B_{\mu}$ definod by $(Q x)(t)=Q(t) x(t)$ is bounded and that $F^{\#}$ has the representation $F^{\#}=F^{*} Q$ where $F^{*}$ is tho adjoint of $F$ considered as - mapping in $B\left(0, B_{\mu}\right)$. Heace, $F^{\# F}=F^{*} Q F \geq 0$. In [24] it is verified that F\#F is Bilbert-Schmidt, so that Theorem 2.2 implies that $\left(I+F^{\#} F\right)$ has the factorization

$$
I+F^{\#} F=\left(I+X^{*}\right)(I+I)
$$

With X carsel.

Now let $\nabla^{*}=\left(I+I^{*}\right)^{-1}-I$. Since $\nabla^{*}$ is Hilbert-Schmidt It has a aatrix kernel $T_{-}(t, s)$. Next define the $Y=N$ matrix valued function $P(t, a)$ on $[-\boldsymbol{x}, \mathrm{T}] \times[-\boldsymbol{x}, \mathrm{T}]$ by 


$$
P(t, a)=\int_{a}^{T} I(t, s) Y(s, a) d \mu(s), a \geq t
$$

where

$$
I(t, s)=F^{\prime}(s, t) Q(s)+\int_{t}^{s} I_{-}(t, \sigma) F^{\prime}(s, \sigma) Q(s) d \sigma
$$

and $Y(t, s)$ defined as in (2.7). The function $P(t, a)$ propidos the foedbeck solution to the regulator problem. This is made precise in the following.

Theorem 2.5. The optimal foodbeck control for (2.1) - (2.2) is given by

$$
\begin{aligned}
\hat{u}(t)= & -P(t, t) \hat{x}(t)-\int_{t}^{\min (T, t+x)} P(t, a) \int_{t-x}^{t} d_{\beta} \eta(\alpha, \beta-a) \hat{x}(\beta) d a \\
& -\int_{t}^{T} P(t, a)\left(\operatorname{BP}^{t} \hat{a}\right)(a) d a
\end{aligned}
$$

where $\hat{x}$ denotes the optinal trajectory, and for each $t$, pt denotes the projection on $\delta,\left(P^{t} z\right)(s)=x[-x, t](s) u(s)$. Furthermore, $P(t, a)$ is square integrable (Lebesgue messure) on both the diagonal and the square [-r,T] $I$ $[-\mathbf{x}, \mathbf{T}]$.

3. CONVERGENCE RESOLTS. The specific optinization problez we shall be considering is the following: 


$$
\min _{n, x} J(\pi, x)=\left\langle x(T), Q_{0} x(T)\right\rangle+\int_{-x}^{T}\langle x(s), Q(s) x(s)\rangle+|z(s)|^{2} d s
$$

subject to the constraint

$$
\begin{array}{ll}
\dot{x}(t)=\int_{-5}^{0} d_{\theta} \eta(t, \theta) x(t+\theta)+\left(B P_{0} \nabla\right)(t) & t \geq 0 \\
x(t)=\phi(t) & t \in[-x, 0],
\end{array}
$$

where $f(\cdot), C\left([-r, 0], B^{N}\right)$ and

$$
(B r)(t)=\sum_{i=0}^{k} x\left[r_{i}-r, T\right](t) B_{i}(t) d\left(t-r_{i}\right)+\int_{t-I}^{t} B(t, \theta) v(\theta) d \theta
$$

The assumptions on $\eta(\cdot, \cdot)$ are the sane as in the precoding section. Te shal1 assune that $\left.\left.\left.0=x_{0}\right\rangle-r_{1}\right\rangle \ldots\right\rangle-x_{k}=-x \operatorname{and} \operatorname{sip}\left|B_{i}(t)\right|=b_{i}\left\langle\infty, \operatorname{sip}_{t, \theta}\right.$ $|B(t, \theta)|=b<\infty$. In the cost (3.1) ve inpose continuity on $Q(s)$.

Interpreting these assumptions in the contert of Section 2 , have $\mu=\lambda+\delta$ vhere $\lambda$ denotes Lebesgue measure, $\delta$ is the Dirac measure ith sopport on $\{T\}$, and $Q(\cdot)$ is aniformly continuous on $[-T, T)$ with $Q(T)=Q_{0}$.

Now consider the following sequence $\left[J_{n}\right.$ \} of epproximations to the cost $\mathbf{J}(\mathbf{n}, \mathbf{x})$ :

$$
\begin{aligned}
J_{n}(x, x) & =\int_{-1}^{T}\langle x(s), Q(s) x(s)\rangle d \mu_{n}(s) \\
& +\int_{-x}^{T}|z(s)|^{2} d s,
\end{aligned}
$$


where $\mu_{n}$ is a sequesce of positive regular Borel measures such that:

B1. $\mu_{n}(T)=1$ for al $1 \mathrm{n}$,

H2. Given $>0$ there exists = such that $n$ ? implies

$$
\left|\mu_{n}[a, b)-\right| b-a||<\in \text { for } a 11=\langle b, a, b=[-x, T] \text {. }
$$

In this section wo Will discrss the convergence properties of the solutions and foedbeck lavs corresponding to the cost approximations above. Henceforth wo refer to the optinization problem with cost (3.1) as problem $\mathscr{P}$. and the problem with cost $(3.5)$ as problex $\mathscr{P}_{n}$. Onless otherwise noted, subscripts eppearing on operators, fonctions, etc. (e.g. F F) will indicate that these terms are associated $\nabla$ ith problem $\mathscr{P}_{n}$.

We begin with the folloring simple resolt.

Lemma 3.1. Let $\mu$ be defined as above and let $\mu_{n}$ satisfy Hu and $H 2$. Then $\mu_{n}-8 \rightarrow \lambda$ (Lebesgue messire) in the * topology of $C^{*}(-I, T)$.

Proof. Choose $>0$ and let $f \in C(-r, T)$. Let $\pi=\left\{t_{i}\right\}_{i=0}^{n}$ be partition of $[-r, T]$ sech that for $|t-s|<|\pi|,|f(t)-f(s)|<\varepsilon$. Then,

$$
\left|\int_{-1}^{T} f d\left(\mu_{n}-\delta\right)-\sum_{1=0}^{n-1} f\left(t_{1}\right) \mu_{n}\left[t_{1}, t_{i+1}\right)\right|<\varepsilon \mu_{n}[-r, T],
$$

and

$$
\left|\int_{-I}^{T} f d s-\sum_{i=0}^{n-1} f\left(t_{1}\right)\left(t_{i+1}-t_{1}\right)\right|<\varepsilon(T+x) .
$$


By H2, $\operatorname{sep}\left|\mu_{j}[-I, T)\right|=k\left\langle\infty\right.$. Now choose as sch that $\left.a^{\prime}\right\rangle$ m implies $\left|H_{a},[a, b)-\right| a-b||<s / a|f|$. Then $1 t$ follows that

$$
\left|\sum_{i=0}^{n-1} f\left(t_{i}\right)\left\{\mu_{m},\left[t_{i}, t_{i+1}\right)-\left(t_{i+1}-t_{i}\right)\right\}\right|<=.
$$

Hence,

$$
1 \int f d\left(\mu_{z^{\prime}}-8\right)-\int f d s \mid<s+2 k \varepsilon .
$$

And the 1 emma is proved./I

Since by definition $F(t, s)=\left[P_{0} B^{*} Y^{\prime}(t, \cdot)\right]^{\prime}(s)(\operatorname{cf}(2.5)-(2.6))$, from (3.4) it follows

$$
F(t, s)=\sum_{i=0}^{k} f_{i}(t, s)
$$

Where

$$
f_{0}(t, s)=I(t, s) B_{0}(s)+\int_{s}^{m i s:-i, s} Y(t, \theta) B(\theta, s) d \theta
$$

and

$$
f_{i}(t, s)=I\left(t, s+x_{1}\right) B_{i}\left(s+I_{1}\right), i=1 \ldots .
$$

Nor let $y=\sup |Y(t, s)|$. Then asing [12, p.149] and the bounds in (3.4) ve have

$$
\operatorname{sip}_{t, s}\left|f_{0}(t, s)\right| \leq \gamma\left(b_{0}+b r\right), \operatorname{sip}_{t, s}\left|f_{i}(t, s)\right| \leq b_{i} \gamma
$$


Also, for $t_{2}>t_{1} \geq=+I_{1}$

$$
\begin{aligned}
\left|f_{0}\left(t_{2}, s\right)-f_{0}\left(t_{1}, s\right)\right| \leq & \exp |m|_{1} \int_{t_{1}}^{t_{2}} m(\sigma) d \sigma\left\{b_{0}+b x\right\} \\
& +b y\left(t_{2}-t_{1}\right) .
\end{aligned}
$$

and

$$
\left|f_{1}\left(t_{2}, s\right)-f_{1}\left(t_{1}, s\right)\right| \leq b_{1} \exp |m|_{1} \int_{t_{1}}^{t_{2}} m(\sigma) d \sigma, i=1, \ldots k
$$

It is not difficalt to show (soe [24]) that $F^{\#}=F^{*} j, F_{z}^{\#}=F^{*} j_{z}$ where $F^{*}$ is the B-space adjoint of F, 1.0. $F^{*}: X^{*} \rightarrow$ O, and $j, j_{n}$ are the mappings of $X$ into $X^{*}$,

$$
\begin{aligned}
& j(x) y=\int\langle y(s), Q(s) x(s)\rangle d \mu(s), \\
& j_{n}(x) y=\int\langle y(s), Q(s) x(s)\rangle d \mu_{n}(s) .
\end{aligned}
$$

Now it follows easily from defintion and the estinates above that $F$ is compact. Thrs, nsing the * - convergence of $f_{n}(x) \rightarrow j(x)$ for each $x$ (from Lemma 3.1), it can then be deduced that $F_{n}^{\#} \rightarrow F^{\#}$ strongly. Consequently from the compactness of $F$ it also follors that $F_{n}^{\#} F \rightarrow F^{\#} F$ uniformly. Noting the form of the open 100 p control 1 ar (in Theorem 2.1), these general considerations are enongh to demonstrate the $L_{2}$ - convergence of the approximate optimal controls and the nniform convergence of the corresponding optimal trajectories resulting from approximations based on $\mathscr{P}_{n^{\circ}}$ Howerer, the anjor ain of this section is to produce the stronger $L_{\infty}$ - convergence of the apporimations for the feedback kernels as well as the controls. and this requires a somerbat more spectfic analysts.

Let $Z$ donote the space $L_{\infty}\left([-r, T], R^{M}\right)$. From the definition of $F(t, s)$ it is evident thet $F^{\#}$ and $F_{n}^{\#}$ are $a 180$ in $B(X, Z)$. Onr first result sharpens the convergence of $F_{n}^{\#} \rightarrow F^{\#}$ discussed above. This resalt (and the method of 
proof) will ford the basis for the $L_{\infty}$ - convergence argaments later.

Leme 3.2. $F_{n}^{\#} \rightarrow F^{\#}$ strong iy in $B(X, Z)$.

Proof. Let $x \in X$. By definition

$$
\left[F^{\#}-F_{n}^{\#}\right] x: \quad t \rightarrow \sum_{i=0}^{k} \int_{i} f_{i}^{\prime}(s, t) Q(s) x(s) d\left(\mu-\mu_{n}\right)(s)
$$

For each i dofine

$$
\tilde{f}_{i}(s, t)= \begin{cases}f_{i}(s, t) & >t+r_{i} \\ f_{i}\left(t+r_{i}, t\right) & s \leq t+r_{i}\end{cases}
$$

Similarly defino

$$
Q_{i}(s, t)= \begin{cases}Q(s) & s>t+r_{i} \\ Q\left(t+r_{i}\right) & s \leq t+r_{i}\end{cases}
$$

and

$$
x_{i}(s, t)= \begin{cases}x(s) & s>t+x_{1} \\ x\left(t+x_{1}\right) & s \leq t+x_{1}\end{cases}
$$

Considerod as families of fonctions parameterized by $t,\left[\tilde{f}_{i}(\cdot, t)\right\}$ is equicontinuous by virtue of $(3.7)-(3.9)$, and $\left\{Q_{i}(\cdot, t)\right\}$ and $\left\{x_{i}(\cdot, t)\right\}$ are equicontinuous by virtue of the uniform continuity of $Q(\cdot)$ and $x(\cdot)$, respectively. Furthermore these families are clearly aniformly bounded. Bence the set

$S=\left\{x_{t} e C\left([-x, T], R^{M}\right): x_{t}(s)=f_{1}(s, t) Q_{i}(s, t) x_{1}(s, t), t,\left[-r, T-x_{1}\right], i=0,1, \ldots t\right\}$ 
is relatively compect in $C\left([-T, T], R^{W}\right)$. Now note thet

$$
\begin{aligned}
\left|\left[F^{\#}-F_{n}^{H}\right](x)(t)\right| & \leq \sum_{i=0}^{k}\left[\left|\int_{[-r, T]} f_{i}(s, t) Q_{i}(s, t) x_{i}(s, t) d\left(\mu-\mu_{n}\right)(s)\right|\right. \\
& \left.+\left|\int_{\left[-x, t+x_{i}\right]} \tilde{f}_{i}(s, t) Q_{i}(s, t) x_{i}(s, t) d\left(\mu-\mu_{n}\right)(s)\right|\right]
\end{aligned}
$$

Dsing the compactiess of $S$ it follows from Lemma 3.1 that the first integral above converges to zero uniformiy with respect to $t$. And since the second integral has constant integrand for each $t$ and $i$, uniform convergence is obtained hero by nsing H2.//

Nor let $H(t, s)$ and $B_{n}(t, s)$ denote the kernels of $F_{F}^{\#}$ and F F respectively. Fubinis thoorem implies

$$
\left|B(t, s)-H_{n}(t, s)\right|=\left|\sum_{L, j} \int f_{i}^{\prime}(\sigma, t) Q(\sigma) f_{j}(\sigma, s) d\left(\mu-\mu_{n}\right)(\sigma)\right| .
$$

Arging as in the previous lemma we can show that $H_{n}(t, s)$ converges uniformly to $B(t, s)$. To see this note that for each fired $i$ and $j$

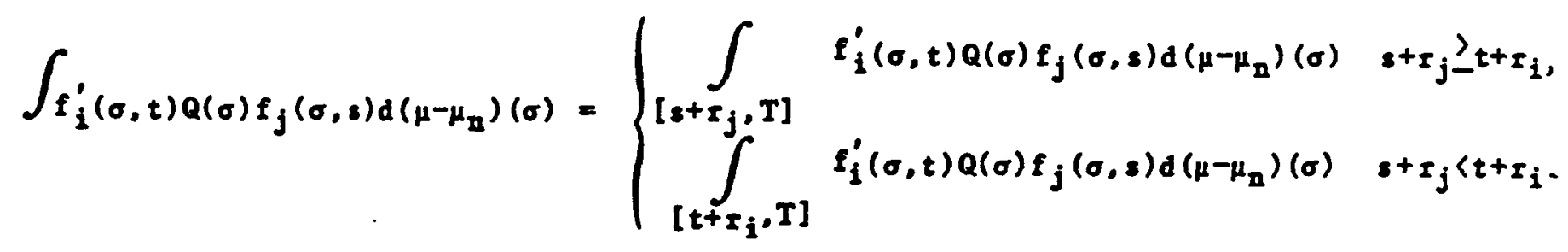

Thos on the set $V=\left\{(s, t): s+x_{j} \geq t+x_{1}\right\}$ we can wite

$$
\begin{aligned}
\int f_{i}^{\prime}(\sigma, t) Q(\sigma) f_{j}(\sigma, s) d\left(\mu-\mu_{n}\right)(\sigma) & =\int_{[-\tau, T]} \tilde{f}_{i}^{\prime}(\sigma, s, t) Q_{j}(\sigma, s) \tilde{f}_{j}(\sigma, s) d\left(\mu-\mu_{n}\right)(\sigma) \\
& -\int_{\left[-I_{,} s+\Sigma_{j}\right]} \tilde{f}_{i}^{\prime}(\sigma, s, t) Q_{j}(\sigma, s) \tilde{f}_{j}(\sigma, s) d\left(\mu-\mu_{n}\right)(\sigma)
\end{aligned}
$$


where $Q_{j}(\sigma, s)$ and $\tilde{f}_{j}(\sigma, s)$ are defined is in Lemma 3.2 and

$$
\tilde{f}_{i}^{\prime}(\sigma, s, t)= \begin{cases}f_{i}^{\prime}(\sigma, t) & 0 \geq s+\Sigma_{j} \\ f_{i}^{\prime}\left(s+\Sigma_{j}, t\right) & \sigma<s+\Sigma_{j}\end{cases}
$$

Dafora convergence of the tategrals parameterizod by $\mathrm{V}$ follors in the manner of the proof of the lemsa. Since this argament also holds for subsets of the form $\left.(s, t): I_{j}<t+x_{1}\right\}$, we obtain the following result.

Leman 3.3. With the notations bove, $B_{n}(t, s) \rightarrow B(t, s)$ nniformig on $[-r, T] x[-x, T]$. In pertienler, $F_{n}^{\#} F \rightarrow F^{\#} F$ in the Hilbert-Schmidt topology (in $B(D)$ ) and in the $B(Z)$ topologg.

To obtain Lo - convergence of the feodback kernels ve will ose a result analogons to the one above regarding the convergence of the kernels of the Volterra factors of $\left(I+F_{n}^{\#} F\right)$. Already we can ose Corollary 2.4 and Lemma 3.3 to obtain Bilbert-Schmidt conpergence of the factors (hence, $\mathrm{L}_{2}$ convergence of theis kernels). But our altimate interest is to demonstrate

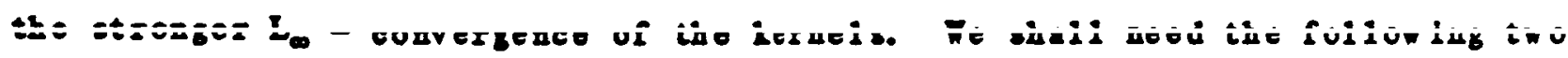
resils which are of some interest in their own right.

Proposition 3.4. Let $X$ be an integral operator on V with essentially

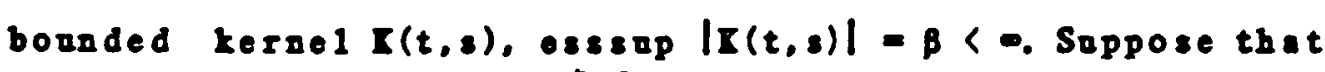

$$
t, 8
$$$$
\operatorname{sep}\left|\left(I+P_{t} \mid P_{t}\right)^{-1}\right|=a<-
$$

$t$

so that $(I+I)$ has the factorization

$$
I+I=\left(I+I^{*}\right)(I+I)
$$

Ith I cansal (cf Thoorem 2.2). Then $\nabla=(I+I)^{-1}-$ I and $\nabla^{*}$ are integral 
operators. With kernels $\prod_{ \pm}(t, s)$ satisfying the bound

$$
\underset{t, s}{\cos \operatorname{sip}}\left|T_{ \pm}(t, s)\right|<p(\alpha, \beta)
$$

where

$$
p(\alpha, \beta)=\beta\left[1+\beta(T+\tau)(1+\alpha \beta(T+\tau))^{2}\right]
$$

Proof. First note that Corollary 2.3 implies $W=(I+I)^{-1}-$ I Ls Bilbert-

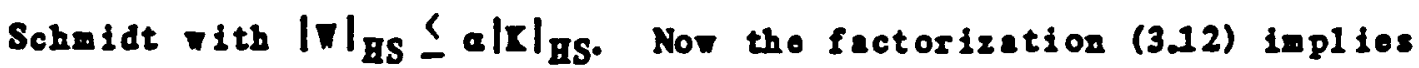

$$
\left(I+I^{*}\right)=(I+I)(I+\pi) \text {. }
$$

Substracting the identity frow the above and applying the projection p_ (1.0. taking anticansal parts) results in for $\theta \geq t$.

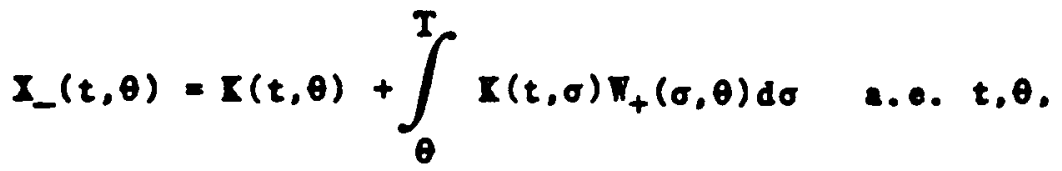

Where $I_{-}(t, \theta)$ and $\nabla_{+}(t, \theta)$ are the kernels of $I^{*}$ and $\mid$ respectively.

Bence for a.e. $t, \theta$,

$$
\left|I_{-}(t, \theta)\right| \leq \beta\left(1+\int_{-\tau}^{T}\left|w_{+}(\sigma, \theta)\right| d \sigma\right) \text {. }
$$

Consequent1y.

$$
\int_{-I}^{T}\left|x_{-}(t, \theta)\right|^{2} d \theta \leq \beta^{2} \int_{-T}^{T}\left[1+\int_{-x}^{T}\left|\sigma_{+}(\sigma, \theta)\right| d \sigma\right)^{2} d \theta
$$




$$
\begin{aligned}
& =\beta^{2}\left[(T+x)+2 \int_{-T}^{T} \int_{-T}^{T}\left|\pi_{+}(\sigma, \theta)\right| d \sigma d \theta+\int_{-T}^{T} \int_{-T}^{T}\left|\pi_{+}(\sigma, \theta)\right| d \sigma 2 d \theta\right. \\
& \leq \beta^{2}(T+x)\left(1+\left|\nabla_{+}\right|_{H S}\right)^{2} \\
& \leq \beta^{2}(T+x)\left(1+a|x|_{\text {HS }}\right)^{2} \\
& \leq \beta^{2}(T+x)[1+\alpha \beta(T+x)]^{2} \quad \text { a.e. } t \text {. }
\end{aligned}
$$

Lot $\tilde{a}=\beta^{2}(T+x)[1+\alpha \beta(T+s)]^{2}$. Then since $\nabla^{*}$ setisfies the identity $\nabla^{*}=-I^{*}-I^{*} \nabla^{*}$, we here

$$
\left|I_{-}(t, \theta)\right| \leq\left|I_{-}(t, \theta)\right|+\int_{-I}^{T}\left|I_{-}(t, \theta) I_{-}(\sigma, \theta)\right| d \theta \quad \text {..e. } t, \theta .
$$

Hence,

$$
\begin{aligned}
& \int_{-I}^{T}\left|I_{-}(t, \theta)\right|^{2} d \theta \leq \int_{-I}^{T}\left|x_{-}(t, \theta)\right|^{2} d \theta+2 \int_{-T}^{T}\left|x_{-}(t, \theta)\right| \int_{-T}^{T}\left|x_{-}(t, s)\right|\left|\pi_{-}(s, \theta)\right| d s d \theta \\
& \left.+\int_{-I}^{T}\left[\int_{-I}^{T}\left|x_{-}(t, s)\right|\left|r_{-}(s, \theta)\right| d s\right]\right]^{2} d \theta \\
& \underline{a}+2 \tilde{a}\left|\nabla_{-}\right|_{\mathrm{HS}}+\tilde{a}\left|\mathbb{Z}_{-}\right|_{\mathrm{HS}}^{2} \\
& \underline{2} a\left(1+a|x|_{\mathrm{BS}}\right)^{2} \quad \text {.... } t
\end{aligned}
$$

Thes,

$$
\underset{t}{\operatorname{cssszp}} \int_{-r}^{T}\left|I_{-}(t, \theta)\right|^{2} d \theta \leq \beta^{2}(T+r)[1+\alpha \beta(T+r)]^{4} .
$$

Now (3.12) elso Inplies

$$
I+I=(I+V)(I+I) \text {. }
$$


Subtracting the identity and epplying $p_{-}$gields

$$
\nabla^{*}+\mathbf{L}_{-}+\left[\boldsymbol{r}^{*} \mathbf{z}\right]_{-}=0
$$

Hence,

$$
\begin{aligned}
\left|I_{-}(t, \theta)\right| & \leq|\mathbf{X}(t, \theta)|+\int_{-x}^{T}\left|T_{-}(t, s)\right||\mathbf{X}(s, \theta)| d s . \\
& \leq \beta\left[1+(T+x)^{1 / 2} \operatorname{ess} \operatorname{sip}_{t}\left[\int_{-T}^{T}\left|T_{-}(t, s)\right|^{2} d s\right]^{1 / 2}\right\}
\end{aligned}
$$

The result follows frow $3.14 . / /$

As the resalt above may be regarded as the Lo anagoe of Corollary 2.3 , the next proposition is the $L_{\infty}$ analogre of Corollerg 2.4. The notations $X$, $\Pi$. $X_{ \pm}(t, s)$ and $W_{ \pm}(t, s)$ will have the same aeaning below as in the precoding proposition.

Proposition 3.5. Let $I$ be as in the proposition above and let $\left[\mathrm{R}_{n}\right]$ denote a sequence of integral operators on 0 -ith essentially bonded kernels $K_{n}(t, s)$ such that

$$
\begin{aligned}
& \text { ess } \operatorname{sip}\left|K_{n}(t, s)\right| \leq 8 . \\
& t, s
\end{aligned}
$$

and

$$
\lim _{\mathrm{n}} \operatorname{ess} \operatorname{sip}_{t, s}\left|\boldsymbol{K}_{\mathrm{n}}(t, s)-\mathbf{I}(t, s)\right|=0
$$

Assome further that $Z_{n} \geq 0$ for each a so that $I+Z_{n}$ hes the factorizetion

$$
I+K_{n}=\left(I+I_{n}^{*}\right)\left(I+I_{n}\right) \quad\left(\text { with } X_{n} \operatorname{couse} 1\right)
$$

Let $\Pi_{n}(t, s)$ denote the kernel of the integral operator $\left(I+Z_{n}\right)^{-1}-I$. 
Then

$$
\underset{n}{\operatorname{im} \cos s \pi p}\left|\pi_{n}(t, s)-\pi_{+}(t, s)\right|=0
$$

Proof. First vite $I+K_{n}=I+I+\left(I_{n}-I\right)$ so that

$$
I+Z_{n}=\left(I+I_{-}\right)\left(I+A_{n}\right)\left(I+I_{+}\right)
$$

where $A_{n}=\left(I+I^{*}\right)^{-1}\left(K_{n}-I\right)(I+I)^{-1}$. By Proposition 3.4 ess sup $\left|\nabla_{ \pm}(t, s)\right| \leq a$ for sone a< $\infty$. Now since $I+Z_{n}$ hes the factorization, so does $I+A_{n}$. Specifical1y, $I+A_{n}=\left(I+Y_{n}^{*}\right)\left(I+Y_{n}\right)$ where $I+Y_{n}=$ $\left(I+I_{n}\right)(I+\pi)$. It then follows from the identity

$$
I+P_{t} A_{n} P_{t}=\left(I+P_{t} Y_{n}^{*} P_{t}\right)\left(I+P_{t} Y_{n} P_{t}\right)
$$

thet

$$
\begin{aligned}
\operatorname{sip}_{t}\left|\left(I+P_{t} A_{n} P_{t}\right)^{-1}\right| & \leq \operatorname{sip}_{t}\left|\left(I+P_{t} Y_{n} P_{t}\right)^{-1}\right|^{2} \\
& \leq\left(1+\left|\Gamma_{n}\right|\right)^{2} \operatorname{sip}\left|\left(I+P_{t} \nabla P_{t}\right)^{-1}\right|^{2} \\
& \leq\left[1+\left|R_{n}\right|_{B S}\right]^{2}\left[\exp \left[1 / 2\left(1+|R|_{B S}^{2}\right)\right]\right]^{2}
\end{aligned}
$$

Bere ve have used Corollary 2.3 to obtain the first tern in the product, and the fact that $\nabla$ is Bilbert-Schaidt and quasinilpotent together ith [7 p. 1039] and Corollary 2.3 to obtain the socond term. Nor define $z_{n}=$ $\left(I+Y_{n}\right)^{-1}-I$ and let $Z_{n}(t, s)$ denote its kernel. Then since

$$
\underset{t, s}{\operatorname{ess} \sup \left|A_{n}(t, s)\right| \leq \beta_{n}\left[1+a(T+r)+a^{2}(T+r)^{2}\right]}
$$

where $\beta_{z}=\operatorname{ess} \operatorname{sip}\left|r(t, s)-I_{n}(t, s)\right|$ and $2 \geq \cos \operatorname{sip}_{t, s}\left|\nabla_{ \pm}(t, s)\right|$.

Proposition 3.4 inplies

$$
\cos \operatorname{sip}_{t, s} Z_{n}(t, s) \leq p\left(\tilde{\beta}_{n}, \tilde{\beta}_{n}\right)
$$


$\nabla$ ith

$$
\tilde{\beta}_{n}=\beta_{n}\left[1+a(T+r)+a^{2}(T+r)^{2}\right]
$$

and

$$
\tilde{a}_{n}=\left[1+\left|\mathbf{I}_{n}\right|_{B S}\right]^{2}\left[\exp \left(1 / 2\left(1+|\mathbf{K}| Z_{\mathrm{BS}}\right)\right]^{2}\right.
$$

Finally, note that

$$
\nabla_{n}=\boldsymbol{\nabla}+\mathbf{z}_{\mathbf{n}}+\boldsymbol{Z}_{\mathbf{n}}
$$

so that

$$
\operatorname{ess} \operatorname{sip}_{t, s}\left|\pi_{n}(t, s)-\pi_{+}(t, s)\right| \leq p\left(\tilde{a}_{n}, \tilde{\beta}_{n}\right)[1+(T+r) a] .
$$

But $p\left(\tilde{a}_{n}, \tilde{\beta}_{n}\right)=0\left(\beta_{n}\right)$. This completes the proof./I

Before proceeding to the main resalt of the soction we will make a digression to establish $L_{\infty}$ convergence of the approxinate control sequence and trajectories. Again we let $Z$ denote the space $L_{\infty}\left([-r, T], R^{M}\right)$. For notational convenience we also introdace the subspec $\Sigma_{0} \subset x$ of initial conditions,

$$
I_{0}=\{x \in I: x(t)=x(0) \text { for } t \geq 0\}
$$

and endor it with the subspace topology.

Theorem 3.6. Let $\mathscr{B}$ denote the nit ball in $C\left([-r, 0], \mathbb{R}^{N}\right)$. For $6 \mathbb{B}$ let $\hat{n}_{n}(\delta)$ and $\hat{n}(d)$ denote the optimal controls for problems $\mathscr{P}_{n}$ and $\mathscr{P}$ respectively. Also let $\hat{x}_{n}(\phi)$ and $\hat{z}(\phi)$ denote the corresponding trajectories. Ther. anif ormly on $\not B$,

$$
\begin{aligned}
& \text { (i) } \quad 1 \operatorname{im}\left|\hat{n}_{n}(\phi)-\hat{n}(\phi)\right|_{Z}=0 . \\
& \text { (ii) } \quad 1 \operatorname{im}\left|\hat{x}_{n}(\phi)-\hat{x}(\phi)\right|_{Z}=0 .
\end{aligned}
$$


Proof. Lot \& $B$. Theorem 2.1 gives the optinal control 1ar es

$$
\hat{n}_{n}(\sigma)=-\left(I+F_{n}^{\#} F\right)^{-1} F_{n}^{\#}(I-L)^{-1} \tilde{\sigma}
$$

where $\tilde{\delta} \cdot x_{0}$ is tho extersion of $\delta$ och that $\tilde{\delta}(t)=\delta(0)$ for $t \geq 0$

Now $(I-L)^{-1 \tilde{b}}$ is recognized as the solution to the homogeneous problem

$$
\dot{x}(t)=\int_{-\pi}^{0} d_{\theta} \eta(t, \theta) x(t+\theta)
$$

Dith initial condition $\phi$. Denoting this solution $x(\phi)$, standerderguments (see [12]) sive

$$
|x(\delta)(t)| \leq|\delta| \exp |\operatorname{m}|_{1}
$$

and

$$
\left|x(\phi)\left(t_{2}\right)-x(\phi)\left(t_{1}\right)\right| \leq|d|\left[\int_{t_{1}}^{t_{2}} \operatorname{m}(s) \mathrm{d} s\right] \exp |\mathrm{m}|_{1}
$$

This the set S,

$$
S=\left\{x \cdot c\left([0, T], R^{N}\right): x(t)=\left[(I-L)^{-1} \tilde{\delta}\right](t) \text { for } \tilde{\delta} \cdot I_{0}-i t h|\tilde{d}| \leq 1\right\}
$$

1s relatively compact in $C\left([0, T], R^{N}\right)$. Now, the triangle inequality gields

$$
\begin{aligned}
\mid \hat{a}_{n} & -\left.\hat{z}\right|_{Z} \leq\left|\left(I+F_{n}^{*} F\right)^{-1}\right|_{B(Z)} \cdot \mid\left(F_{z}^{\#}-F^{\#}\right)(I-L)^{-1} \tilde{d}_{Z} \\
& +\left|\left(I+F_{n}^{\#} F\right)^{-1}-\left(I+F^{\#} F\right)^{-1}\right|_{B(Z)} \cdot\left|F^{\#}(I-L)^{-1}\right|_{Z}
\end{aligned}
$$

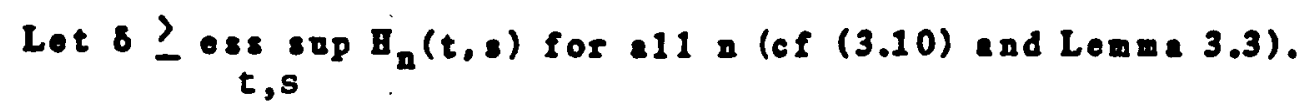


Also let $\nabla_{n}=\left(I+I_{n}\right)^{-1}-I$ there $Z_{n}$ is the carsal factor of $F_{n}^{\#} F$, 1.0 .

$$
\left(I+F_{n}^{\#} F\right)=\left(I+I_{n}^{*}\right)\left(I+I_{n}\right)
$$

Thes.

$$
\left(I+F_{n}^{\#} F\right)^{-1}=\left(I+\nabla_{n}\right)\left(I+\nabla_{n}^{*}\right)
$$

Now let $\nabla_{n}(t, s)$ denote the kernel of $\nabla_{n}$. Thon Proposition 3.4 implies ess sup $\left|w_{n}(t, s)\right| \leq p(1,8)$ independentis of $n$. Hence.

$$
\left|\left(I+F_{n}^{\#} F\right)^{-1}\right|_{B(Z)} \leq[1+p(1, \delta)(T+I)]^{2} \text {, }
$$

and consequentiy

$$
\begin{aligned}
\left|\left(I+F_{n}^{\#} F\right)^{-1}-\left(I+F^{\#} F\right)^{-1}\right|_{B(Z)} \leq\left|\left(I+F_{n}^{\#} F\right)^{-1}\right|_{B(Z)}\left|F^{\#}{ }_{n} F-F^{\#} F\right|_{B(Z)}\left|\left(I+F^{\#} F\right)^{-1}\right|_{B(Z)} \\
\leq[1+\rho(1, \delta)(I+I)]^{4}\left|F_{n}^{\#} F-F^{\#} F\right|_{B(Z)}
\end{aligned}
$$

Te 2180 heve frog $(316)$,

$$
\left|F^{\#}(I-L)^{-1} \tilde{\sigma}\right|_{Z} \leq|\delta|\left|F^{\#}\right|_{B(I, Z)} \cdot \exp |\mathrm{m}|_{1}
$$

By Lomma 3.2, F $\rightarrow F^{\#}$ strongly in $B(X, Z)$. Recall not that $F(t, s)$ (the kernel of $F$ ) vanishes for s ( 0 . Then since $s$ is relatively compect, it f01100s that $\left(F_{n}^{\#}-F^{\#}\right)(I-L)^{-1} \rightarrow 0$ aniformly in $B\left(X_{0}, Z\right)$. This result and Lemma 3.3 together ith (3.19) - (3.21) inserted into (3.18) proves the first assertion of the thoorem.

To prove tho second part let $J_{n}(\phi)=\hat{x}_{n}(\phi)-\hat{x}(\phi)$. Then $J_{n}(\phi)$ satisfies (3.2) Witb zero initial condition and forcing term $B\left(\hat{u}_{n}-\hat{a}\right)$. Therefore for some constant $C$.

$$
\left|y_{n}(\phi)(t)\right|<C \text { oss sop }\left|\hat{n}_{p}(\phi)(t)-\hat{z}(\phi)(t)\right| \text {. }
$$


Thus (ii) follors from (1)./l

Next we present the convergence properties of the sequence of approxinating feedback kernels.

Theorem 3.7. Let $P_{n}(t, a)$ and $P(t, a)$ denote the feedback kernels of Theorea 2.5 associated with problems $\mathscr{P}_{n}$ and $\mathscr{P}$ respectively. Then,

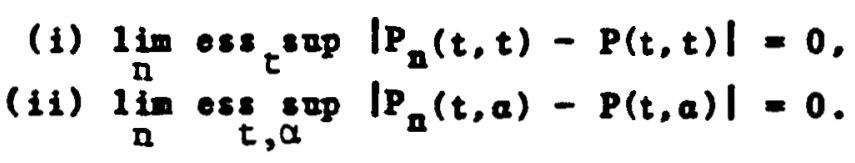

Proof. From (217)-(2.18) ve vite

$$
\begin{aligned}
& P_{n}(t, a)=\sum_{i=0}^{k} \int_{a}^{T} R_{n, i}(t, s) Y(s, a) d \mu_{n}(s), \\
& P(t, a)=\sum_{i=0}^{k} \int_{a}^{T} E_{i}(t, s) Y(s, a) d \mu(s),
\end{aligned}
$$

where

$$
R_{n, i}(t, s)=f_{i}^{\prime}(s, t) Q(s)+\int_{t}^{s-r_{i}} \pi_{n}(t, \theta) f_{i}^{\prime}(s, \theta) Q(s) d \theta
$$

and

$$
K_{i}(t, s)=f_{i}^{\prime}(s, t) Q(s)+\int_{t}^{s-I_{i}} \pi(t, \theta) f_{i}^{\prime}(s, \theta) Q(s) d \theta
$$


Thas,

$$
\begin{aligned}
\left|P_{n}(t, a)-P(t, a)\right| \leq & \sum_{i=0}^{k}\left\{\left|\int_{a}^{T}\left[K_{n, i}(t, s)-\mathbf{K}_{i}(t, s)\right] Y(s, a) d \mu_{n}(s)\right|+\right. \\
& \left.\left|\int_{a}^{T} K_{i}(t, s) Y(s, a) d\left(\mu-\mu_{n}\right)(s)\right|\right\}
\end{aligned}
$$

Nor,

$$
\left|\mathbf{K}_{n, i}(t, s)-\mathbf{K}_{i}(t, s)\right| \leq \int_{t}^{s-I_{i}}\left|\nabla_{n}(t, \theta)-\pi(t, \theta)\right|\left|f_{i}^{\prime}(s, \theta)\right||Q(s)| d \theta
$$

Lemma 3.3 and Proposition 3.5 imply that

$$
\underset{n}{\lim } \operatorname{ess} \operatorname{sip}\left|\pi_{n}(t, \theta)-\pi(t, \theta)\right|=0
$$

And since $\left|f_{i}(s, \theta)\right|$ and $|Q(s)|$ are uniformly bounded, routine argamerts yield a measurable set $Q \subset[-x, T]$ whose complement has zero Lebesgue measure such that

$$
K_{n, i}(t, s) \rightarrow K_{i}(t, s) \text { uniformly on } Q x[-s, T] \text {. }
$$

Using the uniform boundedness of $Y(s, a)$ and the sequence of wessures $\mu_{n}$, it follows that the first integral in (3.22) tends to zero aniformly on $0 \times$ $[-r, T]$. To prove convergence of the second integral in (3.22) ve argae is in Lemma 3.2. Define the family of functions parameterized by a and $\beta$, $\{\tilde{\mathbf{Y}}(\cdot, \alpha, \beta)\}$

$$
\tilde{I}(s, \alpha, \beta)= \begin{cases}Y(s, \alpha) & s \geq \beta \\ Y(\beta, \alpha) & s<\beta,\end{cases}
$$


and the family of functions parameterized by $t$ and $\gamma_{0}\left[\tilde{\mathbf{x}}_{j}\left(t, \gamma_{0} \cdot\right)\right\}$,

$$
\tilde{\mathbf{z}}_{i}(t, \gamma, s)= \begin{cases}\mathbf{I}_{1}(t, s) & \geq y \\ \mathbf{z}_{1}(t, \gamma) & 8<\gamma\end{cases}
$$

It is straightforward to verify using the properties of $Z_{i}(t, s)$ and $Y(s, a)$ that the set

$$
\left\{\tilde{R}_{i}(t, p, \cdot) \tilde{Y}(\cdot, a, p): p=\max \left\{a, t+r_{i}\right\}\right]
$$

1s relative1y compact in $C\left([-r, T], R^{M} I N\right)$. Thas the argament in Lemma 3.2 applies hore to demonstrate that

$$
\underset{n}{\lim } \operatorname{ess}_{t ; \alpha} \operatorname{sip}\left|\int_{a}^{T} \mathbf{K}_{i}(t, s) Y(s, a) d\left(\mu_{n}-\mu\right)(s)\right|=0 .
$$

And the theoren is proved./l

4. APPLICATIONS. In this section ve begin by deriving the optinal feedback kernel essociated with an arbitrary discrete state cost penalty. It $\boldsymbol{\text { ill }}$ be orident that given tho fundemental astrix $Y(t, s)$, the foedbeck kernel in this case can be derived by quadature and antrix inversion. This feodback structure when combined with the results of the preceding section leads to approximetions to the optinal feedback kernel of problem PP (recall (3.1)(3.3)). aiener-Bopf characterization of this kernel, and a priori bounds on Its magnitude.

Lot $v$ denote a positive discrete nessure on $[-Y, T]$ of the form

$$
\int_{f d} v=f\left(s_{n}\right)+\sum_{i=0}^{n-1} s_{1} f\left(s_{1}\right) ; s_{i} \in[-I, T], s_{n}=T
$$


Inserting this measure into $(2.2)$ results in the cost

$$
J(u, x)=\langle x(T), Q(T) \times(T)\rangle+\sum_{i=0}^{n-1} a_{i}\left\langle x\left(s_{i}\right), Q\left(s_{i}\right) \times\left(s_{i}\right)\right\rangle+\underset{-r}{T}|u(s)|^{2} d s
$$

The optinal feedbeck kernel for this cost has the following semiseparable 1 etructure.

Theorem 4.1. Define the matrix functions $G(t)$ and $\bar{Y}(t)$,

$$
\begin{aligned}
& G(t)=\left[\sqrt{z_{0}} F^{\prime}\left(s_{0}, t\right) \vdots---\sqrt{z_{n-1}} F^{\prime}\left(s_{n-1}, t\right) \vdots F^{\prime}\left(s_{n}, t\right)\right]^{\prime} \\
& \bar{Y}(t)=\left[\sqrt{\varepsilon_{0}} Y^{\prime}\left(s_{0}, t\right):---\sqrt{2_{n-1}} Y^{\prime}\left(s_{n-1}, t\right) \vdots Y^{\prime}\left(s_{n}, t\right)\right]^{\prime} .
\end{aligned}
$$

Al so define the antrix

$$
\tilde{Q}=\left[\begin{array}{llll}
Q\left(s_{0}\right) & & \\
& \cdot & & \\
& \cdot & \\
& & \\
& & Q\left(s_{n}\right)
\end{array}\right]
$$

Then the optimal feedback kernel $P(t, a)$ for the problem with dyoamics (2.1) and cost (4.1) can be expressed as

$$
P(t, a)=G^{\prime}(t) \tilde{Q}(I+\delta(t))^{-1} \bar{Y}(a)
$$

where

$$
U(t)=\int_{t}^{T} G(s) G^{\prime}(s) \widetilde{Q d} s
$$


Proof. It is straightforward to verify that the map F\#p resolting from the measure $\checkmark$ in (4.1) is en integral operator with separable kernel $G^{\prime}(t) \tilde{Q G}(s)$. Nor Theorer 2.5 inplies

$$
P(t, a)=\sum_{s_{1}} s_{i} I\left(t, s_{i}\right) Y\left(s_{i}, a\right) .
$$

Bere

$$
Z\left(t, s_{i}\right)=F^{\prime}\left(s_{i}, t\right) Q\left(s_{i}\right)+\int_{t}^{T} T_{-}(t, \sigma) F^{\prime}\left(s_{i}, \sigma\right) Q\left(s_{i}\right) d \sigma
$$

and $\nabla_{-}(t, \sigma)$ donotes the kernel of the enticanal operator $\nabla^{*}$ in the factorization

$$
\left.\left(I+F^{\#} F\right)^{-1}=: I+T\right)\left(I+\nabla^{*}\right)
$$

Dsing the fact that F\# has a separable kernel, it can be verified $[11, p 188]$ that

$$
\nabla_{-}(t, \sigma)=-G^{\prime}(t) \tilde{Q}[I+\nabla(t)]^{-1} G(\sigma)
$$

With $D(t)$ defined as in (4.3). Thes we can wite

$$
\begin{aligned}
P(t, a) & =\sum\left[\sqrt{a}_{i} F^{\prime}\left(s_{i}, t\right) Q\left(s_{i}\right)-G^{\prime}(t) \tilde{Q}[I+\delta(t)]^{-1}\right. \\
& \left.=\int_{t}^{T} G(\sigma) \sqrt{a_{i}} F^{\prime}\left(s_{i}, \sigma\right) Q\left(s_{i}\right) d \sigma\right] \sqrt{a_{i}} Y\left(s_{i}, a\right)
\end{aligned}
$$

Noting the definitions of $G, \bar{Y}$ and 0 , it follows

$$
\begin{aligned}
P(t, a) & =\left[G^{\prime}(t) \tilde{Q}-G^{\prime}(t) \tilde{Q}[I+\delta(t)]^{-1} \delta(t)\right] \bar{Y}(a) \\
& =G^{\prime}(t) \tilde{Q}[I+\delta(t)]^{-1} \bar{I}(a) \cdot / /
\end{aligned}
$$

Note that if in (4.1) wo take for $1 \neq n, a_{1}=0$, and let the operator B 
denote antiplication operator, i.e. $(B r)(t)=B(t) \pi(t)$, then (4.2) reduces Manitins' result for terminal state penalty [20]

\section{T}

$P(t, \alpha)=B^{\prime}(t) Y^{\prime}(T, t) Q(T)\left[I+\int Y(T, \sigma) B(\sigma) B^{\prime}(\sigma) Y^{\prime}(T, \sigma) Q(T) d \sigma\right]^{-1} Y(T, \alpha)$.

Thus Theorem 4.1 can be viered as an extension of this result to problems with control delays and arbitrary discrete sate penal ty.

Nor let $\mu_{n}$ be a sequence of discrete positive messures satisfying $B 1$ and H2, and let $P_{n}(t, a)$ denote the corresponding feedbeck kernels. The theorem inplies each $P_{n}(t, a)$ has the semiseparable form (4.2), while Theorem 3.7 inplies the $L_{\text {o convergence of }} P_{n}(t, a)$ to $P(t, a)(t h$ optimal feedback kernel for problem $\mathscr{P}$ ) and also the $L_{\text {o }}$ convergence of $P_{n}(t, t)$ to $P(t, t)$. Introdacing subscripts in the obvious way, define for oach $n$,

$$
V_{n}(t)=\left[I+D_{n}(t)\right]^{-1}-I
$$

so that the identity

$$
V_{n}(t)=-D_{n}(t)\left[I+\delta_{n}(t)\right]^{-1}
$$

holds. Multiplying by $G_{n}^{0}(t) \tilde{Q}_{n}$ re obtain

$$
\begin{aligned}
G_{n}^{\prime}(t) \tilde{Q}_{n} V_{n}(t) & =-G_{n}^{\prime}(t) \tilde{Q}_{n} \delta_{n}(t)\left[I+\delta_{n}(t)\right]^{-1} \\
& =-G_{n}^{\prime}(t) \tilde{Q}_{n}\left[I+\delta_{n}(t)\right]^{-1}{J_{n}}_{n}(t)
\end{aligned}
$$

Then using the definition of $\nabla_{n}(t)$ and woltiplying by $\bar{Y}_{n}(a)$ it follows that

$$
G_{n}^{\prime}(t) \tilde{Q}_{n} V_{n}(t) \bar{Y}_{n}(a)=\int_{t}^{T} G_{n}^{\prime}(t) \tilde{Q}_{n}\left[I+\sigma_{n}(t)\right]^{-1} G_{n}(\sigma) G_{n}^{\prime}(\sigma) \tilde{Q}_{n} d \sigma \bar{Y}_{n}(a)
$$


Fron (4.2) and the dofinition of $\nabla_{n}(t)$ we have for $a \geq t$

$$
P_{n}(t, a)-G_{n}^{\prime}(t) \tilde{Q}_{n} V_{n}(t) \bar{Y}_{n}(a)=G_{n}^{\prime}(t) \tilde{Q}_{n} \bar{Y}_{n}(a)
$$

And substituting (4.4) into the sbove

$$
P_{n}(t, a)=G_{n}^{\prime}(t) \tilde{Q}_{n} \bar{Y}_{n}(a)-\int_{t}^{T} G_{n}^{\prime}(t) \tilde{Q}_{n}\left[I+D_{n}(t)\right]^{-1} G_{n}(\sigma) G_{n}^{\prime}(\sigma) \tilde{Q}_{n} d \sigma \bar{Y}_{n}(a)
$$

Now lot $P_{n}$ donote the integral operator with kernel $P_{n}(t, a)$. Te then recognize $G_{n}^{\prime}(t) \tilde{Q}_{n}\left[I+\delta_{n}(t)\right]^{-1} G_{n}(\sigma)$, with $\sigma \geq t$, as the kernel of the operator $\left[P_{n} B\right]$. Also $G_{n}^{\prime}(t) \tilde{Q}_{n} \bar{Y}_{n}(a)$ is recognized as the kernel of $F_{n}^{\#} Y_{\text {, where }}{ }_{\text {is }}$ the operator in $B(B, I)$ defined

$$
I_{a}: t \rightarrow \int_{0}^{t} Y(t, s) z(s) d s \text {. }
$$

Thus (4.5) represents the Wiener-Bopf equation

$$
P_{n}=\left(F_{n}^{\#} Y\right)_{-}-\left[\left(P_{n} B\right)-F_{n}^{\#} Y\right]_{-} .
$$

Let $P$ denote the operator with kernel $P(t, a)$. Then since

$$
\lim \left|P_{n}-P\right|_{B S}=0 \text { and } 1 \text { im }\left|F_{n}^{\#} Y-F^{\#} Y\right|_{H S}=0
$$

(where the latter is essentially Lemma 3.3 ), by continaity of the prejection p_ on the space of Bilbert-Schmidt maps ro obtain

$$
P=\left(F^{\#} Y\right)_{-}-\left[(P B) P^{\#} Y\right]_{-}
$$

Te formalize this discossion in the following.

Corollary 4.2. The Tiener-Bopf equation (4.7) has anique BilbertSchmidt solution P. A version of the kerael of $P$ is the optimal feedbeck 
kernel for the optiaization problem $\mathscr{P}(3.1)-(3.2)$. Furthermore this version of the kernel can be approximated in the $L_{\infty}$ topology on the diagonal as well $2 s$ the square by the semiseparable kernels $P_{n}(t, a)$.

Proof. We only need to prove the nniqueness assertion. Suppose there oxist two Hilbert-Schmidt solutions of (4.7) and let 8 denote their difference. Then we obtain

$$
8+\left[(8 B)_{-} \mathrm{F}^{\#} \mathrm{I}\right]_{-}=0
$$

Clearly it is sufficient to show that $(\delta B)_{-}=0$. Define

$$
\tilde{\delta}=-\left[(\delta B)\left[F^{\#} \mathbf{Y}\right]\right.
$$

so that $\tilde{\delta}_{-}=\delta$. Then since $B$ is causal it follows that $(\tilde{\delta} B)_{-}=(\delta B)_{-}$and

$$
\tilde{\delta}+(\tilde{\delta} B)_{-} F^{\#} Y=0
$$

Multiplying by $B$ and noting that $Y B=F(c f(3.6)$ and $(4.6))$

$$
(\tilde{\delta} B)_{-}+\left[(\tilde{\delta} B)_{-} F^{\#} F\right]_{-}=0
$$

We vill show that zero is the only solution to the equation

$$
\mathbf{X}+\left[\mathrm{XF}^{*} \mathrm{~F}\right]_{-}=0
$$

thus proving $(8 B)_{-}=0$, and the result. Now $(4.8)$ is equivalent to

$$
\left[X\left(I+F^{\#} F\right)\right]_{-}=0
$$

with $I$ anticarsal. Since F\#F $\geq 0$, there exists a causal Bilbert-Schmidt map V such thet

$$
I+F^{\#} F=\left(I+\nabla^{*}\right)(I+\nabla)
$$


Thus $I$ solves (4.8) if and only if $Z$ solves

$$
z+[z V]_{-}=0
$$

(These solutions are related by $X=Z\left(I+V^{*}\right)^{-1}$.) Nert consider the mapping $\mathscr{L}$ on the space of Hilbert-Schmidt operators

$$
\mathscr{L}(\mathrm{Z})=[\mathrm{ZV}]_{-}
$$

Then (4.9) is equivalent to $(I+\mathscr{L}) Z=0$. Now by induction we find that

$$
\mathscr{L}^{\mathbf{n}}(\mathbf{Z})=[\mathbf{Z V}]_{-}
$$

so that

$$
\left|\mathscr{L}^{\mathbf{n}}(\mathrm{z})\right| \leq|z|_{\mathrm{BS}}\left|\mathbf{v}^{\mathrm{n}}\right|
$$

But $\nabla$ is qusinilpotent, hence so is $\mathscr{L}$. Thas the onlysolution to (4.9) is $Z=0$, and the theorem is proved./l

We note that the existenco portion of the corollery was proved in a different maner in [24].

When $B$ is a maltiplication operator, (PB) $=$ PB, so that $(4.7)$ becomes

$$
P=\left(F^{\#} \mathbf{Y}\right)_{-}-\left[P B F^{\#} \mathbf{Y}\right]_{-} \cdot
$$

The kernel of F\#Y is easily compted to be of the form $B^{\prime}(t) A(t, s)$ where

$$
A(t, s)=\int_{\max (t, s)}^{T} Y^{\prime}(\sigma, t) Q(\sigma) Y(\sigma, s) d \sigma+Y^{\prime}(T, t) Q(T) Y(T, s) .
$$

Let $A$ denote the operator with kernel $A(t, s)$ and note that $F^{\#} Y=B^{*} A$. Next consider the folloring modification of the viener-Bopf equation (4.7),

$$
\Pi=\Lambda_{-}-\left[\Pi B B^{*} A\right]_{-}
$$


Osing the sane techniques as in the proof of Corollary 4.2 it is possible to show that (4.10) has a noique Hilbert-Schmidt solution $\Pi_{0}$. 1 so note that the corollary implies $B n_{0}=P$. The Wiener-Hopf equation (4.10) is equivalent to the parameterized family of Fredbolm equations Manitios [20] derived via a aeximum principle for obtaining the foodback kernels:

$$
\Pi(t, s)=\Lambda(t, s)-\int_{t}^{T} \Pi(t, \theta) B(\theta) B^{\prime}(\theta) A(\theta, s) d \theta
$$

Corollary 4.2 extends this Wiener-Hopf characterization of the feedback kernel to problems with control delays, end simultaneously provides epproximate solutions.

The special factorization has beon previousiy oxploited in solving Wiener-Hopf equations on finite intervals of the type (410)-(4.11) that arise in inverse problems in the spectral theory of differential operators [8],[18], and in the filtering and smoothing problems for nonstetionery processes [15], [16]. The corollary is in sense solution finding tho right viener-Hopf problem.

Now ve return to the originel problem (3.1) - (3.3) and consider a specific soquence of measures for cenerating approxinations to the optimal foedbeck kernel.

Let $\left[\mu_{n}\right]$ denote the sequence of messures

$$
\int f d \mu_{n}=f(T)+(T+I) / n \sum_{i=0}^{n-1} f(1(T+r) / n-x) .
$$

This sequence is exsily shown to satisfy 11 and H2. Nor suppose the anjorizing function $(t)$ (cf $(2.3)$ ) is bounded and the reighting function $Q(\cdot)$ hes a bonded dorivative. Letting $P_{n}(t, a)$ denote the feedback kernel corresponding to the cost $i$ th messure $\mu_{n}$. it is straightorward (al though 
todions) to derive constant $C$ from the results in Section 3 soch that

$$
\begin{aligned}
& \operatorname{ess} \operatorname{sip}\left|P(t, a)-P_{n}(t, a)\right| \leq c / n, \\
& \text { ess sup } \mid\left(P(t, t)-P_{n}(t, t) \mid \leq c / n .\right.
\end{aligned}
$$

To can also use the epproximations $P_{n}(t, a)$ to obtain an a priori bound on $|P(t, a)|$ in the following $\nabla$ ay.

$$
\begin{aligned}
& \text { First observe that for each } t \\
& \qquad \widetilde{Q}_{n}\left(I+\delta_{n}(t)\right)^{-1} \leq \widetilde{Q}_{n}, n=1,2, \ldots
\end{aligned}
$$

Thes.

$$
\left|P_{n}(t, a)\right| \leq\left|G_{n}^{\prime}(t)\right|\left|\tilde{Q}_{n}\right|\left|\bar{Y}_{n}(a)\right|, \quad n=1,2 \ldots
$$

Where all the norms ebove are operator (matrir) norms on the eppropriate Euclidean spaces. Now for each $a,\left|\bar{Y}_{n}(a)\right|$ is bounded by $\left|\bar{Y}_{n}(a)\right|_{B S}$. (Bere

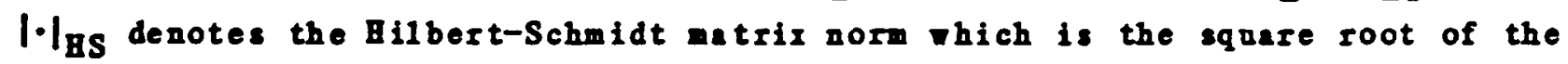
sum of the squares of the matrix entries.) And

$$
\begin{aligned}
& \left|\bar{Y}_{n}(a)\right|_{B S}^{2}=|Y(T, a)|_{\mathrm{ZS}}^{2}+\frac{(I+r)}{n} \sum_{i=0}^{n-1}\left|Y\left(s_{i}, a\right)\right|_{\mathrm{BS}}^{2} \\
& \leq(T+I+1) \times \operatorname{spp}_{S}|Y(s, a)| \text { HS. }
\end{aligned}
$$

A similar bound bolds for $\left|G_{n}^{\prime}(t)\right|$.

$$
\left|G_{n}^{\prime}(t)\right|^{2} \leq(T+I+1) \operatorname{sip}_{S}\left|F^{\prime}(s, t)\right|_{\text {ZS }}^{2}
$$

Thes,

$$
\left|P_{z}(t, a)\right| \leq(T+I+1) \operatorname{sip}_{s}|Q(s)| \cdot \sup _{s}\left|F^{\prime}(s, t)\right|_{B S} \cdot \operatorname{sip}_{s}|I(s, a)|_{B S}
$$


independentis of $n$. And since $P_{n}(t, a) \longrightarrow P(t, a)$ a.e. (on the squere and diegonal), the same bound holds for $|P(t, a)|$.

The $T$ - dependence (the length of the problem interval) in the bound for $|P(t, a)|$ can be expressod differently by noting that

$$
\lim _{n}\left|\bar{Y}_{n}(a)\right|_{\mathrm{BS}}=|\mathrm{Y}(\mathrm{T}, a)|_{\mathrm{ZS}}^{2}+\int_{a}^{T}|Y(s, a)|_{\mathrm{ES}} \mathrm{T} s
$$

And aftex asing the analogors bound on $\left|G_{n}^{\prime}(t)\right|$ wo obtain

$$
\begin{aligned}
& |P(t, a)| \leq \operatorname{sip}_{s}|Q(s)| \cdot\left[|F(T, t)|_{\mathrm{BS}}^{2}+\int_{t}^{T}\left|F^{\prime}(s, t)\right|_{\mathrm{BS}} \mathrm{S}^{\mathrm{d}}\right\}^{1 / 2} \\
& {\left[|Y(T, a)|_{B S}^{2}+\int_{a}^{T}|Y(s, a)|_{B S}^{2} d s\right)^{1 / 2}}
\end{aligned}
$$

When the system (3.1) - (3.4) is time-inverient and stable, a boud independent of $T$ can be established for $|P(t, a)|$. To see this we take $Q(\cdot)=$ Q ( constant matrix) and $\eta(t, \theta)=\eta(\theta)$ where all the roots of the characteristic equetion

$$
\Delta(\lambda)=\lambda I-\int_{-\Sigma}^{0} 0^{\lambda \theta} d \eta(\theta)
$$

ere ssumed to hare negative real part. Also for simplicity we define the operator B es

$$
(B u)(t)=B_{0} u(t)+\int_{t-x}^{t} B(t-\theta) d(\theta) d \theta
$$


with $\left|B_{0}\right|$, sup $|B(t)|$ <b. From the aseomptions on $\eta(\cdot)$ it can bo shown [12] that $Y(t, a)=Y(t-a) \nabla i t h|Y(t)| \leq \exp (-\mu t)$ for somecop $\geq 0$. Thus,

$$
\int_{a}^{T}|Y(s, a)|_{\text {BS }}^{2} \leq \leq \int_{0}^{\infty}|Y(s)|_{\mathrm{BS}} \mathrm{ds}<-.
$$

Since the Laplace transfora $\hat{Y}(\lambda)$ of $Y(t)$ setisfies $Y(\lambda)=\Delta^{-1}(\lambda)$, (see [12]), the inequality above together with Parseral's formule jields

$$
\int_{a}^{T}|Y(s, a)|_{\mathrm{BS}}^{2} \leq 2 \pi \int_{-\infty}^{\infty}\left|\Delta^{-1}(i \lambda)\right|_{\mathrm{BS}}^{2} \mathrm{~d} \lambda
$$

Next note that

$$
\begin{aligned}
\left|F^{\prime}(s, t)\right|_{B S} & \leq b|Y(s-t)|_{B S}+\int_{t}^{\min (s, t+r)}|Y(s-\theta)|_{B S}|B(\theta-t)|_{B S} d \theta \\
& \leq b|Y(s-t)|_{B S}+G(s, t)
\end{aligned}
$$

vhere

$$
G(s, t)=\left\{\begin{array}{l}
b \sqrt{I}\left[\int_{0}^{s-t}|Y(a)|_{H S d a}^{2}\right]^{1 / 2}, t \leq s \leq t+I \\
b \sqrt{I}\left[\int_{t}^{t+I}|Y(s-\theta)|_{\text {HSd }}^{2}\right]^{1 / 2},>t+I
\end{array}\right.
$$

Straightformard approximations then field

$$
\int_{t}^{T}\left|F^{\prime}(s, t)\right|_{B S^{d}}^{2} \leq 2 \pi b^{2}[1+\sqrt{2} r\}^{2} \int_{-\infty}^{\infty}\left|\Delta^{-1}(i \lambda)\right| z S^{2} d \lambda .
$$




$$
|P(t, a)| \leq|a| 2 \pi b(1+\sqrt{2} x) \int_{-\infty}^{\infty}\left|\Delta^{-1}(1 \lambda)\right|_{\text {HS }}^{2} d \lambda
$$

5. ALGORIIHMS. The results that have been presented thus far have emphasized the connections between factorization and the feedback kernels for hereditary systems with control delays, and have not focused on any of the implementation issues concerning the approximation scheme that has been defined. In this Section we will examine in greater detail algorithms based on Theorem 4.1. Particular attention will be paid to time-invariant systems. First, a quick remark about these algorithms in general.

We note that it has already been observed that combining (4.2) with the discretizations (4.12) results in (most cases) an $0(1 / n) L_{\infty}$ convergence of the feedback kernels ${ }^{2}$. This result, which is also valid for time varying systems with control delays, is sharper than the approximations for the feedback operators obtained in [10], [19]. Neither of these articles establishes a priori rates of convergence, nor do the convergence results that are established translate into $\mathrm{L}_{\infty}$ convergence of the kernels. The underlying reason why we are able to obtain the stronger convergence is that we do not approximate the entire semigroup (or evolution operator), but only that piece that is contributed by the fundamental matrix (which we must solve for as a separate computation). In the general time-varying case with discrete state cost at the nodes $\left\{s_{n}\right\}_{i=1}$, this amounts to solving the $n+1$ Volterra equations

$$
Y\left(s_{i}, \sigma\right)=I-\int_{\sigma}^{s_{i}} Y\left(s_{i}, u\right) \eta(u, \sigma-u) d u
$$

In the time-invariant case these computations reduce to the single Volterra equation

$$
Y(t)=I-\int Y(u) \eta(u-t) d u
$$


Once we have the solutions (5.1) or (5.2), the feedback structure (4.2) is straightforward and can be computed from quadrature, matrix inversion and multiplication.

Of course we are not constrained to directly solving (5.1) or (5.2), and we can use other methods for oblaining the fundamental solution - e.g. state approximation methods [2], [3], the method of steps [26], [27] or other available methods for solving Volterra functional differential equations [28], [29], [30]. Having said this, we assume throughout this section that the functions $Y(t, s)$ and $B(t, s)$ have been computed. (A couple of issues associated with these computations will be addressed later.)

One straightforward implementation of (4.2) consists in defining the grid $\left\{s_{i}\right\}_{i=0}^{n}$ so that $s_{i}-s_{i-1}=\Delta=T / n_{1}$ taking $a_{i}=\Delta$, and replacing the integral in (4.3) by a first order Buler quadrature with nodes $\left[s_{i}\right\}$. To see where this leads us, first write $P(t, a)$ in the more symmetrical fashion

$$
P(\tau, \alpha)=G^{\prime}(t) \widetilde{Q}^{1 / 2}\left[I+U^{U}(\tau)\right]^{-1} \widetilde{Q}^{1 / 2} \bar{Y}(\alpha),
$$

where

$$
\underset{\sim}{U}(t)=\tilde{Q}_{\tau}^{1 / 2} \int G(s) G^{\prime}(s) d s \widetilde{Q}^{1 / 2}
$$

Let $\hat{U}\left(s_{i}\right)$ denote the approximation to $\underline{U}\left(s_{i}\right)$,

$$
\hat{U}\left(s_{i}\right)=\sum_{j \geq 1} \Delta \widetilde{Q}^{1 / 2} G\left(s_{j}\right) G^{\prime}\left(s_{j}\right) \widetilde{Q}^{2 / 2},
$$

and form the approximations $\left\{\hat{P}\left(s_{i}, s_{j}\right)\right\}_{j} \geq i$ to $\left\{P\left(s_{i}, s_{j}\right)\right\} j \geq i$

$$
\hat{P}\left(s_{i}, s_{j}\right)=G^{\prime}\left(s_{i}\right) \tilde{Q}^{1 / 2}\left[I+\hat{U}\left(s_{i}\right)\right]^{-1} \widetilde{Q}^{1 / 2} \bar{Y}\left(s_{j}\right)
$$

Now note that

$$
\left[I+\hat{U}\left(s_{i-1}\right)\right]^{-1}=\left[I+\hat{U}\left(s_{i}\right)+\Delta \widetilde{Q}^{1 / 2} G\left(s_{i}\right) G^{\prime}\left(s_{i}\right) \widetilde{Q}^{1 / 2}\right]^{-1}
$$


and that

$$
\operatorname{Rank}\left(\Delta \widetilde{Q}^{1 / 2} G\left(s_{i}\right) G^{\prime}\left(s_{i}\right) \widetilde{Q}^{1 / 2}\right) \leq M
$$

(Recall that $M=$ dimension of the input space.) Thus, using the matrix identity,

$$
\left(X+Y Y^{\prime}\right)^{-1}=X^{-1}-X^{-1} Y\left(Y^{\prime} X^{-1} Y+D^{-1} Y^{\prime} X^{-1}\right.
$$

for compatible matrices $X$ and $Y$, it follows that $\left(I+\hat{U}\left(s_{i-1}\right)\right)^{-1}$ can be updated from $\left(I+\hat{U}\left(s_{i}\right)\right)^{-1}$ in about $2 \mathrm{MNN}^{2} \mathrm{n}^{2}$ operations. Further, exploiting the semiseparable structure of $P(\cdot, \cdot)$, a rough operation count shows that $\left\{\hat{P}\left(s_{i}, s_{j}\right)\right\}_{j} \geq i$ can be computed in approximately $3 \mathrm{MN}^{2} \mathrm{n}^{\prime}$ operations when $\mathrm{M} \leqq N \ll n$.

Considering that there are more than $\mathrm{MNn}^{2} / 2$ values in the matrices $\left\{\hat{P}\left(s_{i}, s_{j}\right)\right\} ; \geq i$, this algorithm is fairly efficient. However, we will subsequently show that it is possible to do substantially better in the time-invariant case. (We will also provide a more complete analysis in this case.)

For the remainder of this section we consider the problem defined by the dynamics

$$
\begin{array}{lll}
\dot{x}(t)=\int_{-r}^{0} d \eta(\theta) x(t+\theta)+\sum_{i=0}^{k} B_{i} u\left(t-r_{i}\right)+\int_{t-r}^{t} B(t-\theta) u(\theta) d \theta, t \geq 0 \\
x(t)=\Phi(t), & t \in[-r, 0] \\
u(t)=0, & t \in[-r, 0]
\end{array}
$$

and cost

$$
J(u, x)=\int_{0}^{T}\langle x(s), Q x(s)\rangle+|u(s)|^{2} d s
$$

The assumptions here are the same as in (9.1) - (3.4), except now everything is time-invariant. (Previously the lower limit in the integral defining the cost ) was taken as -r. This served as a notational expediency in the preceding sections, which we dispense with in the present section.) 
We will explicitly consider the discretizations of $\mathrm{I}$,

$$
J_{n}=\sum_{i=0}^{n-1} \Delta\left\langle x\left(s_{i}\right), Q x\left(s_{i}\right)\right\rangle+\int_{0}^{T}|u(s)|^{2} d s,
$$

where $\left\{s_{i}\right\}_{i=0}^{n}$ is a regular partition of the interval $[0, T]$ with mesh $\Delta=s_{i+1}-s_{i}=I / n$. (Although the mesh points of the partition change with $n$, we will not double subscript the $s_{i}$. This will not lead to any confusion in the sequel.) We will also assume that the point delays in the control, $r_{i}, i=1, \ldots k$, correspond to some subset of the $\left\{s_{i}\right\}, i=0, \ldots . n$.

Again we let $P_{n}(\cdot, \cdot)$ denote the optimal feedback kemel for cost $J_{n}$ and let $P(\cdot, \cdot)$ denote the optimal fecdback kernel for cost $J$. In the time-invariant case Var $\mid n \cdot \cdot) \mid$ and $Q(\cdot)$ are constant, so that (4.13) holds for the sequence $\left\{P_{n}(\cdot, \cdot)\right\}$. The particular algorithm which we will be developing is based on approximately $P_{n}(\cdot, \cdot)$ at the mesh points $\left\{s_{i}\right\}_{i}$ thus it is first necessary to prove that (4.13) actually holds everywhere. Before showing this we need some notations and a couple of simple observations.

For any matrix $M$, as before $|M|_{\text {HS }}$ denotes the Hilbert-Schmidt norm of $M$ (the square root of the sum of the squares of its entries), and for specificity we write $|\mathrm{M}|_{2}$ for the operator norm of $M$ with respect to the corresponding Euclidean metrics. Note that $|\mathrm{M}|_{\mathrm{HS}} \geq|\mathrm{M}|_{2}$.

The fundamental matrix solution $Y(t, a)$ to $(5.4)$ (cf $(2.7)$ ) has the form $Y(t, \alpha)=$ $Y(t-\alpha)$. We set

$$
\begin{gathered}
Y_{Y}=\sup |Y(t)|_{H S^{\prime}} \\
t \in[0, t]
\end{gathered}
$$

and using [12, p. 149] we note that

$$
|Y(t)-Y(s)|_{H S}=O(|t-s|), t, s \in[0, T] \text {. }
$$

Similarly $F(t, s)$ (cf $(3.6)$ ) is a difference kernel, $F(t, s)=F(t-s)$, and using (5.7) and (5.8) we have

$$
\sup _{t \in[0, t]}|F(t)|_{H S}=\gamma_{P}<\infty,
$$

and

$$
|P(t)-P(s)|_{H S}=O(|t-s|), \text { when } 2, \operatorname{se}\left(r_{i}, r_{i+1}\right) \text { for some } i, 0 \leq i \leq k-1
$$

Lemma 5.1. Let $P(t, \alpha)$ denote the optimal feedback kernel for the problem (5.4)(5.5). Then 
$\left|P(t, \alpha)-P\left(t^{\prime}, a^{\prime}\right)\right|=0\left(\left|t-t^{\prime}\right|+\left|\alpha-\alpha^{\prime}\right|\right)$.

Proof. From Theorem 2.5 we have

$$
P(t, \alpha)=\int_{\alpha}^{T} K(t, s) Y(s-\alpha) d s,
$$

where

$$
K(t, s)=F^{\prime}(s-t) Q+\int^{s} W_{-}(t, \sigma) F^{\prime}(s-\sigma) Q d \sigma
$$

$t$

Since $\sup |K(t, s)|<\infty$ (cf Proposition 3.4 and (5.9)), it follows from (5.8) that

$t, 8$

$$
\left|P(t, \alpha)-P\left(t, a^{\prime}\right)\right|<E_{1}\left|\alpha-a^{\prime}\right|
$$

for some constant $K_{1}$ independent of $t, \alpha, \alpha^{\prime}$. Thus using (5.7) it remains to show the existence of a constant $K_{2}$ such that

$$
\int\left|K\left(t_{2}, s\right)-K\left(t_{1}, s\right)\right| d s \leq K_{2}\left|t_{2}-\tau_{1}\right|
$$

Now $F(t)$ has only a finite number of jump discontinuities, so (5.9) and (5.10) imply

$$
\int\left|F\left(s-t_{2}\right)-F\left(s-t_{1}\right)\right| d s=0\left(\left|t_{2}-t_{1}\right|\right)
$$

Hence, it is only necessary to verify that

$$
\int\left|W_{-}\left(t_{2}, \sigma\right)-W_{-}\left(t_{1}, \sigma\right)\right| d \sigma=0\left(\left|t_{2}-t_{1}\right|\right)
$$

To this end let $W^{*}$ denote the operator with kemel $W_{-}(t, \sigma)$ and let $X^{*}=$ $\left(I+W^{*}\right)^{-1}-I$. Denote the kernel of $X *$ by $X(t, s)$. Also let $W$ and $X$ denote the adjoints of $W^{*}$ and $X^{*}$ respectively, with respective kernels $W_{+}(t, s)$ and $X_{+}(t, s)$. Now the factorization (recall Theorem 2.5)

$$
I+E^{*} F=\left(I+X^{*}\right)(I+X)
$$

implies 


$$
X^{*}=\left[F^{*} F\right]_{-}+\left[F^{*} F W\right]_{-}
$$

where $\mathrm{F}^{*} \mathrm{~F}$ has kernel $\mathrm{H}(\mathrm{t}, \mathrm{s})$,

$$
H(t, s)=\int_{\max (t, s)}^{T} F^{\prime}(\sigma-t) Q F(\sigma-s) d \sigma .
$$

Note that

$$
\left|H\left(t_{2}, s\right)-H\left(t_{1}, s\right)\right|=0\left(\left|t_{2}-t_{1}\right|+\left|s_{2}-s_{1}\right|\right)
$$

Now (5.12) is equivalent to

$$
X_{-}(t, s)=H(t, s)+\int H(t, \theta) W_{+}(\theta, s) d \theta \text {, a.e. } s, t .
$$

Because $H(t, s)$ is continuous, $X_{ \pm}(t, s), W_{ \pm}(t, s)$ are also continuous [11], and the equation above holds pointwise. Thus $(5 . \overline{1} 3)$ and the triangle inequality imply

$$
\left.\left|X_{-}\left(t_{2}, 8\right)-X_{-} t_{1}, s\right||=0:| t_{2}-t_{1} \mid\right)
$$

independently of $\mathrm{s}$. Then (5.11) follows from the estimate above and the resolvent identity,

$$
w_{-}(t, s)+X_{-}(\tau, s)+\int_{t}^{s} X_{-}(\tau, \theta) w_{-}(\theta, s) d \theta=0 . / /
$$

Proposition 5.2. sup $\left|P_{n}(t, \alpha)-P(\tau, \alpha)\right|=0(1 / n)$

$$
\tau, \propto \in(0, T)
$$

Proof. Using the identity

$$
A[I+B A]^{-1}=A^{1 / 2}\left[I+A^{1 / 2} B A^{1 / 2}\right]^{-1} A^{1 / 2}
$$

for $A, B \geq 0$, write the feedback kemel (from Theorem 4.1) as,

$$
P_{n}(t, \alpha)=\underline{Z}_{n}^{\prime}(t)\left[I+\Psi_{n}(t)\right]^{-1} \underline{\sim}_{n}(\alpha)
$$


where

$$
\begin{aligned}
& E^{(}(\tau)=\Delta^{1 / 2}\left[F^{\prime}\left(s_{0}-t\right) Q^{1 / 2} \ldots F^{\prime}(T-t) Q^{1 / 2}\right]_{1}^{\prime} \\
& \text { T }
\end{aligned}
$$

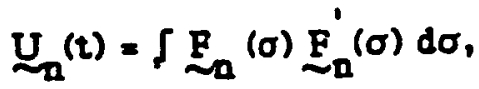

and

$$
Y_{n}(\alpha)=\Delta^{1 / 2}\left[\begin{array}{c}
Q^{1 / 2} Y\left(s_{0}-\alpha\right) \\
\vdots \\
Q^{1 / 2} Y(T-\alpha)
\end{array}\right]
$$

Note the following bounds independent of $\mathrm{n}$ (recall (5.7) and (5.9)):

$$
\sup \left|\Psi_{\mathrm{n}}(\alpha)\right|_{\mathrm{HS}}^{2} \leq \mathrm{I}\left|\mathrm{Q}^{1 / 2}\right|_{\mathrm{HS}}^{2} Y_{\mathrm{Y}}^{2}
$$

$\alpha$

$$
\sup \left|\mathbb{F}_{\mathrm{a}}(t)\right|_{\mathrm{HS}}^{2} \leq I\left|\mathrm{Q}^{1 / 2}\right|_{\mathrm{HS}}^{2} \gamma_{\mathrm{F}}^{2}
$$

$t$

$$
\sup \mid\left(I+U_{n}(t)\right)^{-1} I_{2} \leq 1
$$

$$
\mathbf{t}
$$

Now fix $\left(t_{0}, \alpha_{0}\right) \in(0, I] \times(0, I]$ with $t_{0} \leq a_{0}$. Then there exist indices $i, j$ such that $\left(\tau_{0}, \alpha_{0}\right) \in\left(s_{i-1}, s_{i}\right] \times\left(s_{j-2}, s_{j}\right]$, $i \leq j$. From $(5.8)$ we obtain

$$
\begin{aligned}
& \left.\left|Y_{n}\left(s_{j}\right)-\Psi_{n}\left(\alpha_{0}\right)\right|_{H ~} s_{k \geq j} \leq\left|\sum_{k} \Delta\right| Y\left(s_{k}-s_{j}\right)-\left.Y\left(s_{k}-\alpha_{0}\right)\right|_{H S} ^{2}\right]^{1 / 2}\left|Q^{1 / 2}\right| \\
& \left.=\left|\sum \Delta\right| Y\left(s_{k}-\alpha_{0}+\alpha_{0}-s_{j}\right)-\left.Y\left(s_{k}-\alpha_{0}\right)\right|_{H S} ^{2}\right]^{1 / 2}\left|Q^{1 / 2}\right| \\
& k \geq j \\
& =0(1 / a) .
\end{aligned}
$$

Similarly, expressing $F(\cdot)$ as a sum (as in (\$.6)) and using (5.10) we obtain 


$$
\left|\mathbf{F}_{\mathfrak{n}}\left(s_{\mathbf{i}}\right)-{\underset{\sim}{\mathrm{n}}}_{\mathrm{n}}\left(t_{0}\right)\right|_{H S}=0(1 / \mathrm{n})
$$

Next observe that

$$
\begin{aligned}
\left|\left(I+{\underset{\sim}{n}}_{n}\left(t_{0}\right)\right)^{-1}-\left(I+{\underset{\sim}{n}}_{n}\left(s_{i}\right)\right)^{-2}\right|_{2} & \leq\left|\left(I+{\underset{\sim}{n}}_{n}\left(t_{0}\right)\right)^{-1}\right|_{2}\left|{\underset{\sim}{n}}_{n}\left(t_{0}\right)-{\underset{\sim}{n}}_{n}\left(s_{i}\right)\right|_{H S}\left|\left(I+{\underset{\sim}{n}}_{i}\left(s_{i}\right)\right)^{-1}\right|_{2} \\
& \leq\left|{\underset{\sim}{n}}_{n}\left(t_{0}\right)-\underset{\sim}{U}\left(s_{i}\right)\right|_{H S}
\end{aligned}
$$

But from (5.16) and (5.19) it follows that

$$
\left|U_{n}\left(t_{0}\right)-\Psi_{n}\left(s_{i}\right)\right|_{H S}=0(1 / n)
$$

Putting (5.21) - (5.24) together with the bounds (5.18) - (5.20), and using the triangle inequality we get

$$
\left|P_{n}\left(q_{0}, \alpha_{0}\right)-P_{n}\left(s_{i}, s_{j}\right)\right|_{2}=0(1 / n)
$$

Then using the fact that (4.13) holds for a. e. $t, \alpha$, and that the estimate (5.25) holds for any $\left(i_{0}, \alpha_{0}\right) \in\left(s_{i-1}, s_{i}\right) \times\left(s_{j-1}, s_{j}\right)$, Lemma 5.1 and the triangle inequality imply

$$
\left|P_{n}(t, \alpha)-P(t, a)\right|_{2}=0(1 / n)
$$

for all $t, \alpha \in(0, T] \times(0, T] . / /$

Since the idea behind the algorithm defined in Theorems 5.3 and 5.4 is based on a relatively simple observation that gets somewhat obscured in the notation and proofs of the theorems, it is worthwhile here to brienly remark on this motivating idea.

If we return to the approximate gain defined in (5.3), it turns out that in the time-invariant case each $\hat{U}\left(s_{i}\right)$ is a principal minor of $\hat{U}(0)$. Thus what we would like to 
do is invert $I+\hat{U}(0)$ via a recursion in which all of the principal minors of $I+\hat{U}(0)$ are also inverted. Now by inspection $\hat{U}(0)$ can be identified with the covariance matrix of the random process $\left\{\Delta^{1 / 2} \xi_{i}\right\}$,

$$
\xi_{i}=\Delta \sum_{j=0} Q^{1 / 2} E((i-j) \Delta) \omega_{j}
$$

where $E\left(\omega_{i} \omega_{j}\right)=\delta_{i j} I$. In the signal processing literature (see for example [31]) it is shown that processes of this type admit "fast" filter implementations due to their "near" Toeplitz covariance matrix structure. This is precisely the property we exploit.

Theorem 5.3. Define the symmetric $N(n+1) \times N(n+1)$ mairix $\hat{U}^{n}$ with $N \times N$ block entries $\hat{U}_{\mathbf{i j}}^{\mathfrak{n}}$ where (for $0 \leq \mathbf{j} \leq \mathbf{i} \leq \mathbf{n}$ )

$$
\hat{U}_{i j}^{n}=\sum_{\sigma=0}^{j} \Delta^{2} Q^{1 / 2} E((i-j) \Delta+\sigma \Delta) F^{\prime}(\sigma \Delta) Q^{1 / 2}
$$

Let $Z_{n}$ denote the matrix on $\mathrm{R}^{\mathrm{N}(\mathrm{n}+1)}$,

$$
Z_{n}=\left[\begin{array}{llll}
0 & \cdots & 0 & 1 \\
1 & \ddots & & 0 \\
\vdots & \ddots & & \\
0 & & 1 & 0
\end{array}\right] \text {, }
$$

and for each $p=0,1, \ldots n$, let $\Pi_{n, p}$ denote the projection

$$
\Pi_{p_{1}, p}\left(x_{1} \ldots x_{N(n+1)}\right)^{\prime}=\left(x_{2} \ldots x_{N(p+1)^{\prime}}, 0, \ldots 0\right)^{\prime}
$$

For $p, q=0,1, \ldots n$ define (recall $\Delta=T / n)$

$$
\hat{\mathrm{P}}_{\mathrm{n}}(\Delta \mathrm{p}, \Delta q)=\hat{X}_{\mathrm{n}, \mathrm{n}-\mathrm{p}}^{\prime} \mathrm{Z}_{\mathrm{n}}^{\mathrm{N}(\mathrm{n}+1-\mathrm{p})} \underline{\mathrm{Y}}_{\mathrm{n}}(\Delta q)
$$

where $\underline{\sim}_{\mathrm{n}}$ is defined in (5.17) and

$$
\left.\hat{x}_{n, p}=I I+\Pi_{n, p} \hat{U}^{n} \Pi_{n, p}\right]^{-1}\left[\begin{array}{c}
\Delta^{1 / 2} Q^{1 / 2} F(0) \\
\Delta^{1 / 2} Q^{1 / 2} F(\Delta p) \\
0 \\
0
\end{array}\right]
$$


Then

$\max \left|\hat{P}_{n}(\Delta p, \Delta q)-P(\Delta p, \Delta q)\right|_{2}=O(1 / n)$

$p \leq q$

$p, q \in\{0,1, \ldots . n\}$

Proof. Recall the representation (5.14) for the optimal feedback kernel for cost $\mathbf{I}_{\mathbf{n}}$

$$
P_{n}(t, \alpha)={\underset{\sim}{n}}_{n}^{\prime}(t)\left[I+{\underset{\sim}{n}}_{n}(t)\right]^{-1} \Psi_{n}(\alpha)
$$

Thus we can write

$$
P_{\mathbf{n}}(\Delta p, \Delta q)=X^{\prime}(\Delta p) \underline{\mathbf{Y}}_{\mathbf{n}}(\Delta q)
$$

where

$$
X(\Delta p)=\left[I+U_{n}(\Delta p)\right]^{-1}\left[\begin{array}{c}
0 \\
\Delta^{1 / 2} Q^{1 / 2} P(0) \\
\vdots \\
\Delta^{1 / 2} Q^{1 / 2} P((n-p) \Delta)
\end{array}\right]
$$

Note that $\Psi_{n}(\Delta p)$ is an $N(n+1) \times N(n+1)$ symmetric matrix with $N \times N$ block entires $\mathbb{W}_{i, j}^{n}(\Delta p), \quad i, j=0,1, \ldots n$ where $($ for $0 \leq j \leq i)$

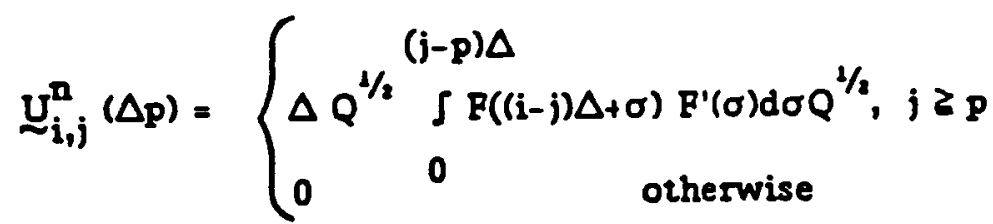

For each $p=0,1, \ldots n$, let

$$
X_{n, p}=\left[I+\Pi_{n, p} U_{n}(0) \Pi_{n, p}\right]^{-1}\left[\begin{array}{c}
\Delta^{1 / 2} Q^{1 / 2} F(0) \\
\Delta^{1 / 2} Q^{1 / 2} F(\Delta p) \\
0 \\
\dot{0}
\end{array}\right]
$$

Observing that

${\underset{u}{i+n-p, j+n-p}}^{n}(\Delta(n-p))=\Psi_{i, j}(0) ; i, j=0,1, \ldots p$, 
it follows from (5.27) and (5.29) that

$$
x(\Delta(n-p))=Z_{n}{ }^{(n-p) N} X_{n, p}
$$

Therefore (using (5.26)),

$$
P_{n}(\Delta p, \Delta q)=X_{n, n-p}^{\prime} Z_{n}^{N(n+1-p)} Y_{n}(\Delta q)
$$

Now,

$$
\left|P_{n}(\Delta p, \Delta q)-P_{n}(\Delta p, \Delta q)\right|_{2} \leq T^{1 / 2}\left|Q^{1 / 2}\right|_{2} Y_{Y}\left|X_{n, n-p}-\hat{X}_{n, n-p}\right|_{2}
$$

(Here we have used (5.18).) But using (5.29) and the definitions of $X$ and $\hat{X}$,

$$
\left.\left|X_{n, n-p}-\hat{x}_{n, n-p}\right|_{2} \leq T^{2 / 2}\left|Q^{3 / 2}\right| Y_{F} \mid \hat{U}^{n}-U_{n}(0)\right)\left.\right|_{H S}
$$

And,

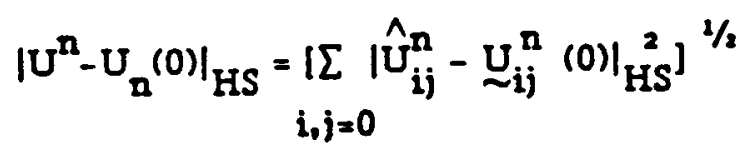

It follows from (5.26) that

$$
\left|\hat{U}_{i j}^{n}-U_{i j}^{n}(0)\right|_{H S}=0\left(\Delta^{2}\right)
$$

independent of $i, j$. Thus the right side of $(5.30)$ is $0(\Delta)$. Hence,

$$
\max \left|P_{n}(\Delta p, \Delta q)-\hat{P}_{n}(\Delta p, \Delta q)\right|_{2}=0(\Delta)
$$

$\mathbf{p}, \mathbf{q}$

The result follows from the estimate above and Proposition 5.2.//

Using the extended LWR algorithm [31] we will next show that $\left\{\hat{\mathrm{X}}_{\mathrm{n}, \mathrm{p}}\right\}_{p=0}^{\mathrm{n}}$ can be computed in a total of $\mathrm{O}\left(\mathrm{n}^{2}\right)$ operations. The theorem above represents the approximate gain in the form

$$
\hat{P}_{n}(\Delta p, \Delta q)=X_{n, n-p}^{\prime} Z_{n}^{N(n+1-p)} Y_{n}(\Delta q)
$$

Now fixing $p$ and letting $q$ vary, these products can be viewed as a convolution. Since convolutions have fast implementations in $0(n \log n)$ operations, it will follow then that $\{\hat{P}(\Delta p, \Delta q)\}_{q \geq p}$ can be computed in $0\left(n^{2} \log n\right)$ operations. 
Theorem 5.4. $\left[\hat{\mathrm{X}}_{\mathrm{n}, \mathrm{p}}\right]_{\mathrm{p}=0}^{\mathrm{n}}$ can be computed in $0\left(\mathrm{n}^{2}\right)$ operations.

Proof. By definition $\hat{\mathbf{x}}_{\mathrm{n}, \mathrm{p}}$ satisfies

$I I+\Pi_{n, p} \hat{U}^{n_{n, p}} \Pi_{n, p}=\Pi_{n, p} \underline{F}_{n}(0)$

We will exhibit a fast recursion for solving (5.31).

A simple calculation shows that

$$
\hat{U}_{i+1, j+1}^{n}-\hat{U}_{i, j}^{n}=\Delta^{2} Q^{1 / 2} F((i+1) \Delta) R^{\prime}((j+1) \Delta) Q^{1 / 2}
$$

Therefore the matrix $\delta\left(\hat{U}^{n}\right)$ with block entries $\hat{U}_{i+1, j+1}^{n}-\hat{U}_{i, j}^{n}, i, j=0,1, \ldots . n-1, \operatorname{can}$ be written

$$
\delta\left(\hat{U}^{2 h}\right)=\Delta^{2}\left[\begin{array}{c}
Q^{1 / 2} F(\Delta) \\
\vdots \\
Q^{2 / 2} \cdot \operatorname{F}(n \Delta)
\end{array}\right]\left[F^{\prime}(\Delta) Q^{1 / 2} \ldots F^{\prime}(n \Delta) Q^{1 / 2}\right]
$$

Consequently, rank $\left(\delta\left(\hat{U}^{n}\right)\right) \leq N \ll$. Eurthermore (and importantly [31]) $\delta\left(\hat{U}^{n}\right.$ ) has a factorization of the form

$$
\delta\left(\hat{U}^{n}\right)=D \Sigma D^{\prime}
$$

with $\mathrm{D}$ an $\mathrm{nNxN}$ matrix and $\Sigma$ an $N \times N$ signature matrix. In fact, this factorization is easily done by inspection:

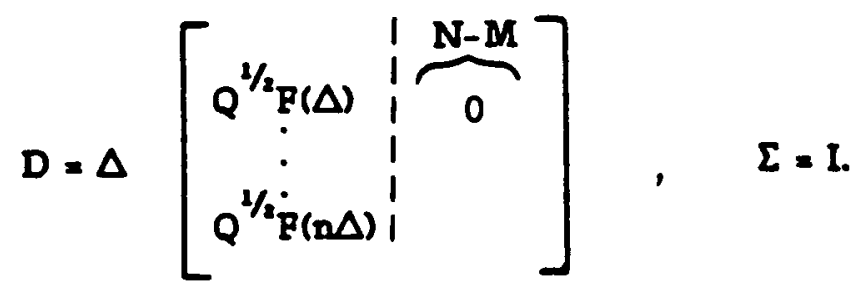


Therefore the LWR algorithm $[31$, p.655] can be used to recursively compute the solutions $M_{p}$ to

$$
\left[I+\Pi_{n, p} \hat{U}^{n} \Pi_{n, p}\right] M_{p}=\left[\begin{array}{c}
\hat{U}_{0, p+1}^{n} \\
\vdots \\
\hat{U}_{p, p+1}^{n} \\
0 \\
0
\end{array}\right] \text {, }
$$

$p=0,1, \ldots n$ in a total of $0\left(N^{3} n^{2}\right)$ operations. With these solutions in hand we can argue as in the scalar Toeplitz case (see for example [32]) to recursively solve the system (5.31). These details are supplied below.

So now consider solving the problem

$$
\left[I+\Pi_{n, p} \hat{U}^{n} \Pi_{n, p}\right] \hat{x}_{n, p}=\Pi_{n, p} \underset{\sim}{R}(0),
$$

given $\hat{\mathrm{x}}_{\mathrm{n}, \mathrm{p}-1}$ and $\mathrm{M}_{\mathrm{p}-1}$ (from (5.32)).

Let $F_{p}$ denote the $N(p+1) \times M$ matrix composed of the first $N(p+1)$ rows of $E_{n}(0)$, and let $f_{p}$ denote the $N x M$ matrix composed of the $N p+1$ through $N(p+1)$ rows of $\mathbb{F}_{n}(0)$. Thus we can write

$$
P_{p+1}=\left[\begin{array}{l}
p_{p} \\
f_{p}
\end{array}\right]
$$

Let $R_{p}$ denote the $N(p+1) \times N(p+1)$ matrix composed of the northwest corner of $I+\hat{U}^{n}$, and let $I_{p}$ denote the $N \times N$ southeast corner of $R_{p}$. Also let $\underset{\sim p}{M} \in R^{N(p+1) x N}$ denote the matrix consisting of the first $N(p+1)$ rows of $M_{p_{A}}$ and similarly let $N_{p}$ denote the $N(p+1) x M$ marrix consisting of the first $N(p+1)$ rows of $x_{n, p}$. 
Now define

$$
u_{p+1}=\left[\begin{array}{c}
\hat{U}_{0, p+1}^{n} \\
\vdots \\
\hat{U}_{p, p+1}^{p}
\end{array}\right] \text {, }
$$

and consider the equation

$$
\left[\begin{array}{ll}
R_{p} & v_{p+1} \\
u_{p+1}^{\prime} & r_{p+1}
\end{array}\right]\left[\begin{array}{l}
\mu \\
v
\end{array}\right]=\left[\begin{array}{l}
F_{p} \\
f_{p}
\end{array}\right]
$$

Note that

$$
\left[\begin{array}{l}
\boldsymbol{\mu} \\
v
\end{array}\right]=\mathbf{N}_{\mathbf{p + 1}}
$$

From the top of the equations in (5.34) we obtain

$$
\mathcal{L}=R_{p}^{-1} F_{p}-R_{p}^{-1} v_{p+1} v=N_{p}-\mathbb{M}_{p} v .
$$

Substituting this into the bottom equation, we get

$$
\left[r_{p+1}-u_{p+1}^{\prime} \mathbf{M}_{p}\right] v=f_{p}-u_{p+1} N_{p}
$$

Noting that

$$
0<\left[\begin{array}{cc}
I & 0 \\
-M_{p}^{\prime} & L_{N \times N}
\end{array}\right]\left[\begin{array}{cc}
R_{p} & u_{p+1} \\
u_{p+1}^{\prime} & \tau_{p+1}
\end{array}\right]\left[\begin{array}{cc}
1 & -M_{p} \\
0 & L_{N x N}
\end{array}\right]
$$




$$
=\left[\begin{array}{cc}
R_{p} & 0 \\
0 & r_{p+1}-M_{p}^{\prime} u_{p+1}
\end{array}\right]
$$

$\left(I_{N x N}=N \times N\right.$ identity matrix), it follows

$$
v=\left[r_{p+1}-u_{p+1}^{\prime} M_{p}\right]^{-1}\left[f_{p}-u_{p+1}^{\prime} N_{p}\right\}
$$

Thus, given $M_{p}$ and $N_{p}, N_{p+1}$ can be computed from (5.36) and (5.37) in $O\left(N^{2} p\right)$ -operations $\left(p \gg N\right.$ ). Hence, in particular $\left\{\hat{x}_{n, p}\right\}_{p=0}^{n}$ in $(5.31)$ can be computed in a recursive manner in a total of $O\left(n^{2}\right)$ operations.//

In [33] infinite dimensional Chundrasekhar equations are derived for time-invariant hereditary systems withour control delay. A fast algorithm based on approximating the Chandrasekhar equations is obtained and is shown to possess the same convergence properties as reported in [10]. Although there are no direct connections between the algorithm we developed here and the one in [33], there are some general connections between Chandrasekhar equations and the inversion of near Toeplitz systems [31].

We note that stability of the algorithms of this section with respect to the data $Y(t), F(t)$ is easily demonstrated. For suppose $Y(\cdot)$ and $P(\cdot)$ were replaced by $Y_{E}(\cdot)$ and $F_{E}(\cdot)$ with

$$
\sup _{t \in[0, T]}\left|Y(t)-Y_{c}(t)\right|,\left|F(t)-P_{c}(t)\right|<\varepsilon .
$$

Using the obvious notation, we obtain the corresponding error estimates,

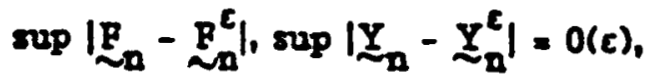

$$
\begin{aligned}
& t
\end{aligned}
$$

and

$$
\max \mid \hat{U}_{i j}^{n}-\hat{U}_{i j}^{n, \varepsilon} q_{H S}=0(\varepsilon) \Delta
$$


all independent of $\mathrm{n}$. Substituting these errors into the appropriate places in Theorem 5.3, it follows that an $O(\varepsilon)$ perturbation in the data $Y(t), F(t)$ yields an $O(\varepsilon)$ perturbation in the estimate of the feedback kemel (all of these estimates in the sup norm).

Another feature to note is that although the implementation defined by Theorem 5.3 and Theorem 5.4 is recursive backward in time for the computation of the feedback kernel (just as one would suspect - e.g. the Riccati equation is solved backward in time), in terms of the algorithm's utilization of the fundamental matrix $Y(t)$, it is actually forward in time. Thus any approximating scheme for the computation of $Y(t)$ can be readily incorporated into the algorithm. This remark is also true for the implementation of Theorem 4.1 introduced in the beginning of this section.

One final remark concerning the algorithm is that in the event that the point delays $\left\{r_{i}\right\}_{i=1}^{k}$ do not correspond to a subset of the nodes $\left\{s_{i}\right\}_{i=0}^{n}$, the estimate in (5.22) is only $O(1 / \sqrt{n})$. Thus the estimate in Theorem 5.3 would also be $0(1 / \sqrt{n})$. Of course Theorem 4.1 has the flexibility to place nodes anywhere, and it may be possible to recover the stronger convergence by considering algorithms arising from different discretization strategies.

We conclude this section with the following simple scalar example:

$$
\min J(u, x)=\int^{2}|x(t)|^{2}+|u(t)|^{2} d t
$$

0

subject to the constraint

$$
\dot{x}(\tau)=x(t)+x(t-1)+n(t)
$$

Since we are seeking the optimal feedback kernel $P(t, \alpha)$, it is not necessary to prescribe an initial condition above.

The lgorithm described in the beginning of the section based on the approximation (5.3) and the recursive inversion of $I+\hat{U}\left(s_{i}\right)$ via the "matrix inversion 
lemma", was programmed using a few lines of Bortran code. Because the fundamental solution $Y(t)$ to the differential equation is easily derived on the interval $[0,2]$, the exact solution was used in the algorithm. (Recall that an $O(\varepsilon)$ error in the approximation of $Y(\cdot)$ results in an $O(\varepsilon)$ error in $P(t, a)$.)

Discretizations with mesh width $\Delta=.025, .01, .005$, were considered. The results for this problem coincided fairly well with the theory. We observed essentially linear (uniform) convergence of the feedback kernels as predicted. Tables 5.1 - 5.3 contain these results. Table 5.4 contains values of the kernel obtained via the Riccati equation approach using linear spline approximations of the history space. The gain computed using these approximations appeared to have converged to two (and in some instances three) significant figures. The author is indebted to Professor I. S. Gibson for providing these values for comparison.

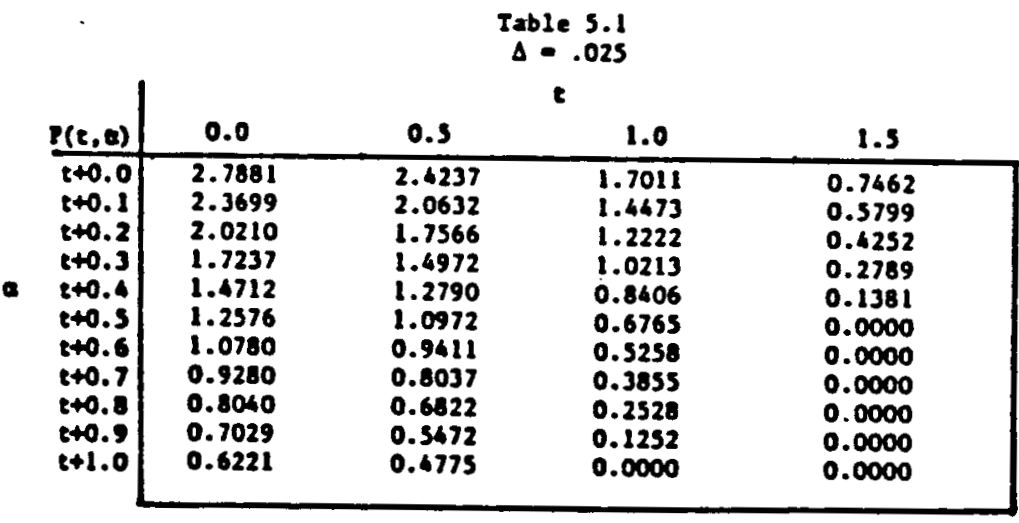

\begin{tabular}{|c|c|c|c|c|}
\hline$P(t, 0)$ & 0.0 & 0.3 & 1.0 & 1.3 \\
\hline $\begin{array}{l}t+0.0 \\
t+0.1 \\
t+0.2 \\
t+0.3 \\
t+0.4 \\
t+0.5 \\
t+0.6 \\
t+0.7 \\
t+0.8 \\
t+0.5 \\
t+1.0\end{array}$ & $\begin{array}{l}2.7300 \\
2.3279 \\
1.9844 \\
1.6919 \\
1.4335 \\
1.2335 \\
1.0570 \\
0.9096 \\
0.7878 \\
0.6886 \\
0.6093\end{array}$ & $\begin{array}{l}2.3951 \\
2.0378 \\
1.7361 \\
1.4770 \\
1.2609 \\
1.0806 \\
0.9270 \\
0.7916 \\
0.6721 \\
0.3659 \\
0.4710\end{array}$ & $\begin{array}{l}1.6963 \\
1.4110 \\
1.2165 \\
1.0162 \\
0.8361 \\
0.6727 \\
9.5228 \\
0.3832 \\
0.2513 \\
0.1244 \\
0.0000\end{array}$ & $\begin{array}{l}0.7521 \\
0.3845 \\
0.4285 \\
0.2810 \\
0.1391 \\
0.0000 \\
0.0000 \\
0.0000 \\
0.0000 \\
0.0000 \\
0.0000\end{array}$ \\
\hline
\end{tabular}


Table 3.3

$\Delta \cdot .005$

\begin{tabular}{|c|c|c|c|c|}
\hline \multirow[b]{2}{*}{$P(t, a)$} & \multicolumn{4}{|c|}{$t$} \\
\hline & 0.0 & 0.5 & 1.0 & 1.5 \\
\hline $\begin{array}{l}t+0.0 \\
t \neq 0.1 \\
t+0.2 \\
t+0.3 \\
t+0.4 \\
t+0.5 \\
t+0.6\end{array}$ & $\begin{array}{l}2.7140 \\
2.3140 \\
1.9724 \\
1.6814 \\
1.4364 \\
1.2556 \\
1.0500\end{array}$ & $\begin{array}{l}2.3855 \\
2.0293 \\
1.2265 \\
1.4703 \\
1.2549 \\
1.0753 \\
0.9223\end{array}$ & $\begin{array}{l}1.6919 \\
1.4388 \\
1.2145 \\
1.0144 \\
0.8346 \\
0.6714 \\
0.5217\end{array}$ & $\begin{array}{l}0.7541 \\
0.3859 \\
0.4295 \\
0.2817 \\
0.1394 \\
0.0000 \\
0.0000\end{array}$ \\
\hline $\begin{array}{l}t+0.7 \\
t+0.8 \\
t+0.9 \\
t+1.0\end{array}$ & $\begin{array}{l}0.9036 \\
0.7825 \\
0.6839 \\
0.6051\end{array}$ & $\begin{array}{l}0.7876 \\
0.6887 \\
0.5631 \\
0.4688\end{array}$ & $\begin{array}{l}0.3824 \\
0.2508 \\
0.1241 \\
0.0000\end{array}$ & $\begin{array}{l}0.0000 \\
0.0000 \\
0.0000 \\
0.0000\end{array}$ \\
\hline
\end{tabular}

Table 5.4

Spline Approximation

\begin{tabular}{|c|c|c|c|c|}
\hline \multirow[b]{2}{*}{$P(t, 0)$} & \multicolumn{4}{|c|}{$\begin{array}{c}\text { Spline Approximation } \\
\qquad\end{array}$} \\
\hline & 0.0 & 0.5 & 1.0 & 1.5 \\
\hline $\begin{array}{l}t+0.0 \\
t+0.1 \\
t+0.2 \\
t+0.3 \\
t \neq 0.4 \\
t \neq 0.5 \\
t \neq 0.6 \\
t \neq 0.7 \\
t+0.8 \\
t+0.9 \\
t+1.0\end{array}$ & $\begin{array}{l}2.6915 \\
2.2986 \\
1.9639 \\
1.6769 \\
1.4263 \\
1.2114 \\
1.0463 \\
0.8994 \\
0.2725 \\
0.6786 \\
0.6013\end{array}$ & $\begin{array}{l}2.3742 \\
2.0177 \\
1.7215 \\
1.4685 \\
1.2489 \\
1.0605 \\
0.9234 \\
0.7862 \\
0.6620 \\
0.5567 \\
0.4690\end{array}$ & $\begin{array}{l}1.6860 \\
1.4355 \\
1.2165 \\
1.0150 \\
0.8284 \\
0.6658 \\
0.3230 \\
0.3881 \\
0.2404 \\
0.1079 \\
0.0323\end{array}$ & $\begin{array}{l}0.7491 \\
0.5894 \\
0.4366 \\
0.2776 \\
0.1257 \\
0.0217 \\
0.0005 \\
0.0032 \\
0.0005 \\
0.0004 \\
0.0000\end{array}$ \\
\hline
\end{tabular}


CONCLODING REMARTS. Oex focrs has beed on the control problem for the RFDE on

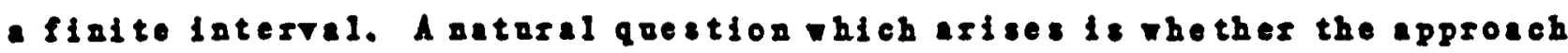
cas be adapted to treat tho lifintte tine problem. Soreral aspects of tho enalysis can probably be exterded to this case. (Sone of these extensions appear sontrivial horererl. For example, al though the factorization resules and projection lategrals discrssed la Soction 2 are based on Hilbert-Schmidt assamptioss (which aze not ralid for problens on the semi-infinite interval), for stable time-iaviriant ojstems the infinite-time factoriration counterpert to (2.16) is the elaselcal Niener-Bopf factorisation. Thes ve expect that the optinel control lar can at least be formulated in the sane ananes in Thoores 2.5 for the Infinite-tine problem. Ang approxination schene devised thereafter roold nocessasily heve to consider epproziasting solutions to a Wiener-Bopf equetion. In epifit soch an approsch aight wake contact vith some vork of Deris [4].

Fundanental to the anajsis of this report wastho exploftetion of tro properties of the factorization approsh of [24]. First, that the entire semigroup never needs approxination, and second that connections betroen the

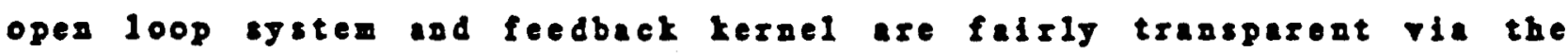
fectorization. These properties are not wique to the BFDE control problem, and as a final comant we remerk that the coseral approach should lead to epproxinate feedback lars in other settings as vell. 


\section{POOTNOTES}

1. The kernel $I(t, s)$ of an integral operator $I$ is semiseparable tf there exist aetrix functions $B_{1}(t)$ and $G_{1}(s), 1=1,2$, exch that $I(t, s)=B_{1}(t) G_{1}(s)$ for s $<t$, and $X(t, s)=\mathrm{H}_{2}(t) G_{2}(s)$ for $\geq t$. The kernel. is separable $1 f$ v0 can choose $E_{1}=B_{2}$ and $G_{1}=G_{2}$. In this case the essociated operator $I$ bas finite rank.

2. It Is unfortangte that although thls convergence ls obtained ising first-order quadrare ochere, it is sot a priorl ovident that emplojing a higher order schere rould result in improved corvergence. The stubling block is that the conversence andysis ro heve used is based on properties of the fundamental natrix, which is seneraliy only absolutely contingons. and thos preclodes ang straightorvard extensions. 


\section{ACKNOWLEDGEMENT}

The author wishes to thank Professor Alan Schumitzky and Professor J. S. Gibson for some very fruitful discussions on the subject of this report.

\section{RERERENCES}

1. I. T. Banks. Represedtation for Solutions of Linenr Punctional Equations, I. Diff. Eq 5. (1969), pp. 399-409.

2. I. T. Benks and J. A. Burns. Bereditary Control Probleas: Numerical Methods Based on Averaging Approximations. Sin I Control Optimization, 16. (1978), Pp. 169-208.

3. I. T. Benks end I. G. Roser, Splize Approrinations for Linesz Nonertonomors Delay Systems, I, Yeth Anale Apel. 96. (1983), Pp. 226-268.

4. J. B. Devis, Tiener-Bopf Metbods for Open-loop Dnstablo Distributed Systems. SIAY I Control Optimization. 17. (1979), pp. 713-728.

5. . C. Delfort, The Linear Quadratic Optial Control Problez for Bereditary Differential Systems: Theory and Namerical Solution. Apele Yethe Optes 3. (1977), pp. 101-162.

6. H. C. Delforr, The Linear Qadratic Optial Codtrol Problez vith Delays in the State and Control Variables: A Stato Space Approach, Certre de Recherche de Hatheratiques Appliquees, Dafrersity of Montreal, CRMA-1012. 2981.

7. N. Donford and J. T. Schwertz, Linear Operators. Part II. IilerIntersclence, Ner Iork. 1963.

8. I. H. Gelfend end B. M. Lerdtan, On tho Deteralastion of a Diferential Equetion from tts Spectral Fanction, AMS Trenslations. Series 2. (1955). PP. $253-304$. 
9. J. S. Gibson. The Riccati Integral Equtlons for Optinal Control Problens on Bilbert Space. SIAN I Control Optimizition. 17, (1979), pp. 537-565.

10. J. 8. Gibsor, Ilnear-quadratic Optinal Control of Bereditery Differential Systems: Infinite Dimensional Riccati Equations and Nomerical Approximations. SIAY J Control Optimization. 21, (1983). pp. 95-139.

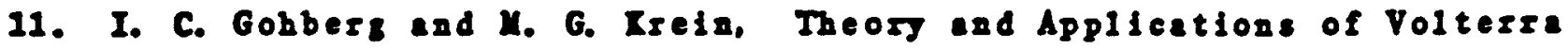
Operators in Blibert Space, MS. Propiderce, R.I.. 1970.'

12. J. Helo, Theory of Punctional Differeatial Equations, Springer-Verles, N.Y.. 1977.

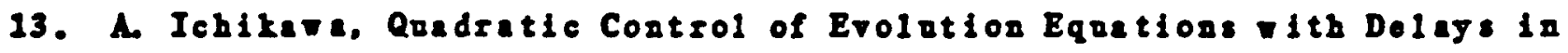
Contro1. SIAM J, Control Optimization, 20, (1982), PP. 645-668.

14. I. Ito, Regulator Problez for Bereditary Differential Systems vitb Control Delags. ICASE Report 82-3, NASA Lasieg Resench Conter, Bampton. VA. 1982 .

15. T. Iallath and P. Frost. An Innorations Approach to Least Squares Estiantion. Part II: Lidear Smoothing in Additive Thite Noiso, IEEE Irense Ant, Control, AC-13, (1968), Pp. 655-660.

16. T. Iatlath. A Note on Lest Squeres Estimation by the Innovations Method, SIAY I. Control, 10, (1972), pp. 477-486.

17. H. N. Koiro and E. B. Lee, Controlier Synthesis for Linear Systems ith Rotarded State and Control Variables and Quadratic Cost, Artomeica, 8, (1972). Pp. 203-208.

18. M. G. Irein, On Inverso Problezs for a Nonbomogeneods Cord. Dok1. Aked. Nenk SSSR, 82, (1952).

19. I. Inalsch. Approziantion Scheres for the Linesr-Quadratic Optimal Control Problea Ascociated Wth Deleg Equations. SIAN I. Control Optimization, 20. (1982), pp. 506-540. 
20. A. Manitius, Optimal Control of Linear Time-lag Processes with Quadratic Performance Indexes, in Proc. of Fourth IFAC Congress, Warsaw, Poland, (1969), pp. 16-28.

21. A. McNabb and A. Schumitzky, Factorization of Operators-II: Initial Value Methods for Linear Two Point Boundary Value Problems, L. Math Anal. Appl., 31, (1970), pp. 391-405.

22. M. Milman and A. Schumitzky, On a Class of Operators on Hilbert Space with Applications to Factorization and Systems Theory, L. Math. Anal. Appl., 99, (1984), pp. 494-512.

23. M. Milman, Special Factorization and Riccati Integral Equations, I. Math. Anal. Appl., 100, (1984), pp. 155-187.

34. M. Milman, J. Foster and A. Schumitzky, Optimal Feedback Control of Infinite Dimensional Linear Systems, I. Math. Anal. Appl. (to appear)

25. R. B. Vinter and R. H. Kwong, The Infinite Quadratic Control for Linear Systems with State and Control Delays: An Evolution Equation Approach, SIAM L. Control Optimization, 19, (1981), pp. 139-153.

26. R. Bellman, On the Computational Solution of Differential-Difference Equations, L. Math. Anal. Appl., 2, (1961), pp. 108-110.

27. R. Bellman and K. L. Cooke, On the Computational Solution of a Class of Functional Differential Equations, L. Math. Anal. Appl., 12 (1965), Pp. 495-500.

28. H. J. Oberle and H. J. Pesch, Numerical Treatment of Delay Differential Equations by Hermite Interpolation, Numer. Math, 97, (1981), pp. 235-255.

29. C. W. Cryer and L. Tavemini, The Numerical Solution of Vollerra Functional Differential Equations by Euler's Method, SIAM I. Numer. Anal., 9, (1972), pp. 105-129. 
30. A. Feldstein and R. Goodman, Numerical Solution of Ordinary and Retarded Differential Equations with Discontinuous Derivatives, Numer. Math. 21, (1973), pp. 1-13).

31. B. Friedlander, T. Kailath, M. Morf, and L. Ljung, Extended Levinson and Chandrasekhar Equations for General Discrete-Time Linear Estimation Problems, IEEE Trans. Aut. Contr., AC-23, (1978) pp. 653-659.

32. G. H. Golub and C. P. Van Loan, "Matrix Computations", The Johns Hopkins University Press, Baltimore, Maryland, 1983.

33. J. A. Burns, K. Ito, and R. K. Powers, Chandrasekhar Equations and Computational Algorithms for Distributed Parameter Systems, Proceedings of the 23 rd CDC, Las Vegas, NV, Dec. 1984. 
TECHNICAL REPORT STANDARD TITLE PAGE

\begin{tabular}{|c|c|c|}
\hline 1. Report No. 87-6 & 2. Government Accossion No. & 3. Recipient's Catalog No. \\
\hline \multirow{2}{*}{\multicolumn{2}{|c|}{$\begin{array}{l}\text { 4. Title and Subtitle } \\
\text { Approximating the Linear Quadratic Optimal } \\
\text { Control Law for Hereditary Systems With } \\
\text { Delays in the Control }\end{array}$}} & $\begin{array}{l}\text { 5. Report Date } \\
\text { March 15, } 1987\end{array}$ \\
\hline & & 6. Porforming Organization Code \\
\hline \multicolumn{2}{|c|}{ 7. Author(s) ${ }_{\text {Mark H. Milman }}$} & 8. Performing Orgonizotion Report No. \\
\hline \multirow{3}{*}{\multicolumn{2}{|c|}{$\begin{array}{l}\text { 9. Performing Orgonization Nome and Address } \\
\text { JET PROPULSION LABORATORY } \\
\text { California Institute of Technology } \\
\text { 4800 Oak Grove Drive } \\
\text { Pasadena, California } 91109\end{array}$}} & 10. Work Unit No. \\
\hline & & $\begin{array}{c}\text { 11. Contract or Grant No. } \\
\text { NAS7-918 }\end{array}$ \\
\hline & & 13. Type of Report and Period Covered \\
\hline \multirow{2}{*}{\multicolumn{2}{|c|}{$\begin{array}{l}\text { 12. Sponsoring Agency Name and Address } \\
\text { NATIONAL AERONAUTICS AND SPACE ADMINISTRATION } \\
\text { Washington, D.C. } 20546\end{array}$}} & JPL $P$ L \\
\hline & & $\begin{array}{l}\text { 14. Sponsoring Agency Code } \\
\text { RE156 BK-506-46-11-03-00 }\end{array}$ \\
\hline
\end{tabular}

15. Supplementary Notes

16. Abstract

The fundamental control synthesis issue of establishing a priori convergence rates of approximation schemes for feedback controllers for a class of distributed parameter systems is addressed within the context of hereditary systems. Specifically, a factorization approach is presented for deriving approximations to the optimal feedback gains for the linear regulatorquadratic cost problem associated with time-varying functional differential equations with control delays. The approach is based on a discretization of the state penalty which leads to a simple structure for the feedback control law. General properties of the Volterra factors of Hilbert-Schmidt operators are then used to obtain convergence results for the controls, trajectories and feedback kernels. Two algorithms are derived from the basic approximation scheme, including a fast algorithm, in the time-invariant case. A numerical example is also considered.

17. Key Words (Selected by Author(s))

Numerical Analysis

Systems Analysis
18. Distribution Statement

Unclassified -- Unlimited
19. Security Classif. (of this report) Unclassified
20. Security Classif. (of this page) Unclassified
21. No. of Poges 69
22. Price 